\title{
Kolmogorov problem on widths asymptotics and pluripotential theory
}

\author{
Vyacheslav Zakharyuta
}

Abstract. Given a compact set $K$ in an open set $D$ on a Stein manifold $\Omega, \operatorname{dim} \Omega=n$, the set $A_{K}^{D}$ of all restrictions of functions, analytic in $D$ with absolute value bounded by 1 , is a compact subset of $C(K)$. The problem on the strict asymptotics for Kolmogorov diameters (widths) :

$$
-\ln d_{s}\left(A_{K}^{D}\right) \sim \sigma s^{1 / n}, s \rightarrow \infty .
$$

was stated by Kolmogorov in an equivalent formulation for $\varepsilon$-entropy of that set $[\mathbf{K} \mathbf{1}, \mathbf{K 2}, \mathbf{K T}]$. It was conjectured in $[\mathbf{Z 6}, \mathbf{Z 7}]$ that for "good" pairs $(K, D)$ such an asymptotics holds with the constant $\sigma=2 \pi\left(\frac{n !}{C(K, D)}\right)^{1 / n}$, where $C(K, D)$ is the pluricapacity of the "pluricondenser" $(K, D)$, introduced by Bedford-Taylor [BT2]. In the one-dimensional case it is equivalent to Kolmogorov's conjecture about the $\varepsilon$-entropy of the set $A_{K}^{D}$, which has been confirmed by efforts of many authors (Erokhin, Babenko, Zahariuta, Levin-Tikhomirov, Widom, Nguyen, Skiba - Zahariuta, Fisher - Miccheli, et al).

In $[\mathbf{Z 6}, \mathbf{Z 7}]$ the above problem had been reduced (the proof was only sketched there) to a certain problem of pluripotential theory about approximating the relative Green pluripotential of the "pluricondenser" $(K, D)$ by pluripotentials with finite set of logarithmic singulatities. The latter problem has been solved recently by Nivoche $[\mathbf{N 1}, \mathbf{N} 2]$ and Poletsky $[\mathbf{P}]$. Here we give a detailed proof of the above-mentioned reduction, which provides, together with the Nivoche-Poletsky result, a positive solution of our conjecture about asymptotics of Kolmogorov diameters.

\section{Introduction}

Let $K$ be a compact set in an open set $D$ on a Stein manifold $\Omega, H^{\infty}(D)$ the Banach space of all bounded and analytic in $D$ functions with the uniform norm, and $A_{K}^{D}$ be a compact subset in the space of continuous functions $C(K)$ consisted of all restrictions of functions from the unit ball $\mathbb{B}_{H^{\infty}(D)}$; since it will be always assumed that the restriction operator is injective, one may infer that $A_{K}^{D}$ $=\mathbb{B}_{H^{\infty}(D)}$.

2000 Mathematics Subject Classification. Primary 41A46; 32A; 32U; Secondary 32Q28; 46A04; 46E20.

Key words and phrases. Widths asymptotics, pluripotential theory, spaces of analytic functions, extendible bases, Hilbert scales. 
Given a compact set $A$ in a metric space $X$ we denote by $N_{\varepsilon}(A)$ the smallest integer $N$ such that $A$ can be covered by $N$ sets of diameter not greater than $2 \varepsilon$. Following $[\mathbf{M}]$ we define the $\varepsilon$-entropy of $A$ by the formula: $H_{\varepsilon}(A):=\ln N_{\varepsilon}(A)$ (notice that the information theory $\varepsilon$-entropy $\left[\log _{2} N_{\varepsilon}(A)\right]+1$ is asymptotically equivalent to $H_{\varepsilon}(A) / \ln 2$ as $\left.\varepsilon \rightarrow 0\right)$. Kolmogorov raised the problem about strict asymptotics $([\mathbf{K} 1, \mathbf{K} 2, \mathbf{T} 1, \mathbf{V}, \mathbf{K T}])$

$$
H_{\varepsilon}\left(A_{K}^{D}\right) \sim \tau\left(\ln \frac{1}{\varepsilon}\right)^{n+1}, \varepsilon \rightarrow 0
$$

with some constant $\tau$ (the weak asymptotics for $H_{\varepsilon}\left(A_{K}^{D}\right)$ had been already proved by him under some natural restrictions on $K$ and $D$ ).

For a set $A$ in a Banach space $X$ the Kolmogorov diameters (or widths) of $A$ with respect to the unit ball $\mathbb{B}_{X}$ of the space $X$ are the numbers (see,e.g., [T2]):

$$
d_{i}\left(A, \mathbb{B}_{X}\right):=\inf _{L \in \mathcal{L}_{i}} \sup _{x \in A} \inf _{y \in L}\|x-y\|_{X},
$$

where $\mathcal{L}_{i}$ is the set of all $i$-dimensional subspaces of $X$. For a pair of normed spaces $Y \hookrightarrow X$ with a linear continuous imbedding we shall write simply $d_{i}(Y, X)$ instead of $d_{i}\left(\mathbb{B}_{Y}, \mathbb{B}_{X}\right)$; in particular, $d_{i}\left(A_{K}^{D}\right):=d_{i}\left(H^{\infty}(D), A C(K)\right)$, where $A C(K)$ is the completion of the set of all traces of functions, analytic on $K$, in the space $C(K)$.

From Mityagin $[\mathbf{M}]$ and Levin-Tikhomirov $[\mathbf{L T}]$ it follows that the asymptotics (1.1) is equivalent to the following asymptotics for Kolmogorov diameters of the set $A_{K}^{D}$ :

$$
\ln d_{i}\left(A_{K}^{D}\right) \sim-\sigma i^{1 / n}, i \rightarrow \infty .
$$

with the constant $\sigma=\left(\frac{2}{(n+1) \tau}\right)^{1 / n}$.

Kolmogorov conjectured that, in the case $n=1$, the constant $\tau$ coincides with the Green capacity $\tau(K, D)$ for proper pairs $(K, D)$. Recall that the Green capacity of a condenser $(K, D)$ on a Riemann surface $\Omega$ is the number $\tau(K, D):=\frac{1}{2 \pi} \int \Delta \omega$, where $\omega(z)=\omega(D, K ; z)$ is the generalized Green potential, defined by the formula

$$
\omega(z):=\limsup _{\zeta \rightarrow z} \sup \left\{u(\zeta): u \in S h(D),\left.u\right|_{K} \leq 0, u(\zeta)<1 \text { in } D\right\},
$$

here $\operatorname{Sh}(D)$ stands for the class of all subharmonic functions in $D$ and $\Delta \omega$ is understood as a positive Borel measure (supported by $K$ ).

Kolmogorov's hypothesis has been confirmed by efforts of many authors ([B, E1, E2, Z1, LT, Ng, W, ZS, FM]). The following statement summarizes, in a sense, those one-dimensional results.

Proposition 1.1. Let $K$ be a non-polar compact subset of an open set $D$ on an open one-dimensional Riemann surface $\Omega, K=\widehat{K}_{D}$, and $D$ is a relatively compact open set in $\Omega$ with boundary $\partial D$ consisting of a countable set of compact connected components at least one of which has more than one point. Then the asymptotics (1.3) holds with $n=1$ and $\sigma=\frac{1}{\tau(K, D)}$.

An important tool in the proof of those results (see, e.g., $[\mathbf{Z S}]$ ) is the classical fact of potential theory about the approximation of the potential (1.4) by finite combinations $\sum_{k=1}^{m} \alpha_{k} g_{D}\left(\zeta_{k}, z\right)$, where $g_{D}(\zeta, z)$ is the Green function of $D$ with the unit logarithmic singularity at $\zeta$. 
The asymptotics (1.3) for $n \geq 2$ was known for a long time only in some particular cases (see, e.g.,[KT, V, Z3, ARZ]).

In $[\mathbf{Z 6}]$ (see, also $[\mathbf{Z 7}]$ ) it was conjectured that for a good enough pair $K \subset$ $D$ on a Stein manifold $\Omega, \operatorname{dim} \Omega=n$, the asymptotics (1.3) holds with $\sigma=$ $2 \pi\left(\frac{n !}{C(K, D)}\right)^{1 / n}$, where $C(K, D)$ is the pluricapacity of the "pluricondenser" $(K, D)$, introduced by Bedford-Taylor [BT2]. It was sketched in $[\mathbf{Z 6}]$ how to reduce the problem about the asymptotics (1.3) for $n \geq 2$ to the certain problem of pluripotential theory (suggested as an analogue of the above one-dimensional fact). We state it (see Problem 1.2 below) after some necessary definitions.

The Green pluripotential $\omega(z)=\omega(D, K ; z)$ of a pluricondenser $(K, D)$ on a Stein manifold $\Omega$ is defined by the same formula (1.4) with the class $P \operatorname{sh}(D)$ of all plurisubharmonic functions in $D$ instead of $S h(D)$ in it. We say that $(K, D)$ is a pluriregular pair on $\Omega$ provided the conditions: (a) $K$ is a compact subset of an open set $D \subset \Omega$ such that $K$ coincides with its holomorphic envelope $\widehat{K}_{D}$ with respect to $D$ and the set $D$ has no component disjoint with $K$; (b) $\omega(D, K ; z) \equiv 0$ on $K$ and $\lim _{z \rightarrow \partial D} \omega(D, K ; z)=1$, that is $\omega\left(D, K ; z_{j}\right) \rightarrow 1$ for any sequence $\left\{z_{j}\right\}$ $\subset D$ having no limit point in $D$. Given $F=\left\{\zeta_{1}, \ldots, \zeta_{\mu}, \ldots, \zeta_{m}\right\} \subset D$ and $\alpha=$ $\left(\alpha_{\mu}\right) \in \mathbb{R}_{+}^{m}$ the Green multipole plurisubharmonic function $g_{D}(F, \alpha ; z)$ is defined $([\mathbf{Z 6}, \mathbf{K l}, \mathbf{L e}, \mathbf{Z 7}])$ as a regularized upper envelope of the family of all functions $u \in P \operatorname{sh}(D)$, negative in $D$ and satisfying the estimate $u(z) \leq \alpha_{\mu} \ln \left|t\left(\zeta_{\mu}\right)-t(z)\right|+$ const in some neighborhood $U_{\mu}$ of each point $\zeta_{\mu}$ in local coordinates $t: U_{\mu} \rightarrow \mathbb{C}^{n}$; see below in section 5 about this function more in detail. The following problem was posed in $[\mathbf{Z 6}, \mathbf{Z 7}]$.

Problem 1.2. Given a pluriregular pair $(K, D)$ on a Stein manifold does there exist a sequence of multipole Green functions $g_{D}\left(F^{(j)}, \alpha^{(j)} ; z\right)$ converging to $\omega(D, K ; z)-1$ uniformly on any compact subset of $D \backslash K$ ?

This problem has been solved recently by Nivoche $[\mathbf{N 1}, \mathbf{N 2}]$ and Poletsky $[\mathbf{P}]$ (see below Proposition 8.1), which covers an important part of the final positive proof of our conjecture on Kolmogorov problem.

In this paper we represent a detailed proof of the reduction part which was only sketched in the survey $[\mathbf{Z 7}]$ (somewhat more comprehensive proof from $[\mathbf{Z 6}]$ has been never published).

It is natural to modify the Kolmogorov problem in the following more general way. Denote by $A(D)$ the Fréchet space of all functions analytic in $D$ with the topology of uniform convergence on compact subsets and by $A(K)$ the locally convex space of all germs of analytic functions on $K$ with the usual inductive limit topology. We are concerned with the strict asymptotics of the sequence of Kolmogorov diameters $d_{i}\left(X_{1}, X_{0}\right)$ of the unit ball of a Banach space $X_{1}$ with respect to the unit ball of a Banach space $X_{0}$ for couples of Banach spaces $X_{0}, X_{1}$ satisfying the linear continuous imbeddings:

$$
X_{1} \hookrightarrow A(D) \hookrightarrow A(K) \hookrightarrow X_{0}
$$

and closely related with the spaces $A(D)$ and $A(K)$ in the following sense.

Definition 1.3. We say that a couple of Banach spaces $X_{0}, X_{1}$ satisfying the imbeddings (1.5) is admissible for a pair $(K, D)$ if for any other couple of Banach 
spaces $Y_{0}, Y_{1}$ satisfying the linear continuous imbeddings:

$$
X_{1} \hookrightarrow Y_{1} \hookrightarrow A(D) \hookrightarrow A(K) \hookrightarrow Y_{0} \hookrightarrow X_{0},
$$

we have $\ln d_{i}\left(Y_{1}, Y_{0}\right) \sim \ln d_{i}\left(X_{1}, X_{0}\right)$ as $i \rightarrow \infty$.

For any pluriregular pair $(K, D)$ there exists an admissible couple $X_{0}, X_{1}$ (see below, Corollary 4.10) and the asymptotic class of the sequence $\ln d_{i}\left(X_{1}, X_{0}\right)$ is rather a characteristic of the pair $(K, D)$, than of any individual couple $X_{0}, X_{1}$ admissible for this pair. Moreover, since the spaces $A(K)$ and $A(D)$ are nuclear, there are admissible couples of Hilbert spaces, which allows to apply the Hilbert scale technics.

Problem 1.4. ([Z6, Z7]) Let $(K, D)$ be a pluriregular pair "compact set-open set" on a Stein manifold $\Omega$. Does the strict asymptotics

$$
\ln d_{i}\left(X_{1}, X_{0}\right) \sim-2 \pi\left(\frac{n ! i}{C(K, D)}\right)^{1 / n}, i \rightarrow \infty
$$

hold for some (hence, for any) couple of Banach spaces $X_{0}, X_{1}$, admissible for $(K, D)$ ?

Developing our approach from $[\mathbf{Z 6}, \mathbf{Z 7}]$ and applying the above-mentioned result of Nivoche-Poletsky we shall give the positive solution of this problem, namely the following theorem will be proved in Section 9 after substantial preparatory considerations in sections 2-8.

Theorem 1.5. Let $K$ be a compact set on a Stein manifold $\Omega$ and $(K, \Omega)$ be a pluriregular pair. Then the asymptotics (1.6) holds for any couple of Banach spaces $X_{0}, X_{1}$, admissible for $(K, \Omega)$.

REMARK 1.6. The statement of this theorem remains true assuming that the pair $(K, \Omega)$ satisfies all conditions in the definition of a pluriregular pair besides that $K$ is supposed to be only non-pluripolar (instead of the condition $\omega(\Omega, K ; z) \equiv 0$ on $K)$. But in what follows, for the simplicity sake, we will consider only pluriregular pairs.

As a consequence we obtain an answer to the question about the asymptotics (1.3) specifying the fuzzy terms "good enough" or "proper" in the above conjectures.

Corollary 1.7. Given a pluriregular pair $(K, D)$ the asymptotics

$$
\ln d_{i}\left(A_{K}^{D}\right) \sim-2 \pi\left(\frac{n ! i}{C(K, D)}\right)^{1 / n}, i \rightarrow \infty .
$$

holds if and only if the couple $\left(A C(K), H^{\infty}(D)\right)$ is admissible for $(K, D)$.

The last statement, though being final, is too general and calls for some concrete description of admissibility. We discuss some necessary and sufficient conditions of admissibility of a couple $\left(A C(K), H^{\infty}(D)\right)$ in the sections 4,9. On the other hand, we consider in Section 9 certain classes of pluriregular pairs $(K, D)$, for which the $\operatorname{asymptotics}(1.3)$ does not hold with the constant $\sigma=2 \pi\left(\frac{n !}{C(K, D)}\right)^{1 / n}$ but may be true with some larger constant. 


\section{Preliminaries}

Notation. For a pair of positive sequences we write $a_{i} \prec b_{i}$ if there is a constant $C$ such that $a_{i} \leq C b_{i}$. If $X$ and $Y$ are locally convex spaces, then $X \hookrightarrow Y$ stands for a linear continuous imbedding. We use the notation: $|x|_{F}:=\sup \{|x(z)|: z \in F\}$. Given a Banach space $X$ the notation $\mathbb{B}_{X}$ is for its closed unit ball.

Some facts of Pluripotential Theory. In what follows $\Omega$ is a Stein manifold, $\operatorname{dim} \Omega=n$. The set of all plurisubharmonic functions in an open set $D \subset \Omega$ is denoted by $P \operatorname{sh}(D) ; M P(D)$ stands for the set of all maximal plurisubharmonic functions in $D$. Let us remind that $u \in P \operatorname{sh}(D)$ is maximal in $D$ if for any subdomain $G \Subset D$ and for each function $v \in P \operatorname{sh}(D)$ it follows that the inequality $v(z) \leq u(z)$ takes place on $G$ provided that it is valid on the boundary $\partial G$. A Stein manifold $\Omega$ is called pluriregular (or hyperconvex) if there is a negative function $u \in P \operatorname{sh}(\Omega)$ such that $\lim u\left(z_{j}\right)=0$ for any sequence $\left\{z_{j}\right\}$ having no limit point in $\Omega$. An open set $D \Subset \Omega$ is strongly pluriregular if there is an open set $G \ni D$ and a function $u \in C(G) \cap P \operatorname{sh}(G)$ such that $D=\{z \in G: u(z)<0\}$.

The Green pluripotential of a condenser $(K, D)$ on $\Omega$ (that is $K$ is a compact set in an open set $D \subset \Omega$ ) is the function

$$
\omega(z)=\omega(D, K ; z):=\limsup _{\zeta \rightarrow z} \sup \{u(\zeta): u \in P(K, D)\},
$$

where $P(K, D)$ is the set of all $u \in P \operatorname{sh}(D)$ such that $\left.u\right|_{K} \leq 0$ and $u(\zeta)<1$ in $D$. The following two families of sublevel sets are important for further considerations:

$$
D_{\alpha}:=\{z \in D: \omega(z)<\alpha\}, \quad K_{\alpha}:=\{z \in D: \omega(z) \leq \alpha\}, 0<\alpha<1 .
$$

A compact set $K \subset \Omega$ is pluriregular if $\omega(D, K ; z) \equiv 0$ on $K$ for any open set $D \supset K$. We say that a pair (condenser) $(K, D)$ is pluriregular if (a) both $K$ and $D$ are pluriregular; (b) $\widehat{K}_{D}=K$; (c) $D$ has no components disjoint with $K$. It is known that $\omega(D, K ; z)$ is continuous in $D$ for a pluriregular condenser (see, e.g. $[\mathbf{Z 3}, \mathbf{W l}])$.

Due to Bedford and Taylor [BT1, BT2] (inspired by [CLN]), the MongeAmpére operator $u \rightarrow\left(d d^{c} u\right)^{n}$ is well-defined as an operator from the space $L^{\infty}(\Omega, l o c) \cap$ $\operatorname{Psh}(\Omega)$ to the space $\mathcal{M}(\Omega)$ of non-negative Borel measures with the weak convergence topology; it is "continuous" with respect to monotone sequences of functions; therewith this operator is continuous as an operator from the space $C(\Omega) \cap P \operatorname{sh}(D)$ to the space $\mathcal{M}(\Omega)$. Maximality of a function $u \in P \operatorname{sh}(\Omega) \cap L^{\infty}(\Omega, l o c)$ in $D \subset \Omega$ is equivalent to $\left(d d^{c} u\right)^{n}=0$ in $D$; in particular, $\left(d d^{c} \omega(D, K ; z)\right)^{n} \equiv 0$ in $D \backslash K$ for a pluriregular pair $(K, D)([\mathbf{B T} \mathbf{1}, \mathbf{S a 1}])$.

Of prime importance for our considerations is the notion of pluricapacity of a condenser $(K, D)$, which in the case of a pluriregular condenser can be written in the form $([\mathbf{B T 2}])$ :

$$
C(K, D):=\int_{K}\left(d d^{c} \omega(D, K ; z)\right)^{n} .
$$

For facts from Pluripotential Theory which are not explained here (or below) we send the reader to the book $[\mathbf{K l}]$ warning only that our notation may differ from used there.

Spaces. Let $D$ be an open set on a Stein manifold $\Omega$. Denote by $A(D)$ the Fréchet space of all functions analytic in $D$ with the topology of locally uniform 
(or compact) convergence on $D$, determined by the sequence of seminorms

$$
\|x\|_{s}:=|x|_{K_{s}}=\max \left\{|x(z)|: z \in K_{s}\right\}, \quad x \in A(D), s \in \mathbb{N},
$$

where $K_{s}$ is any non-decreasing sequence of compact subsets exhausting $D$. By $A(K)$ we denote the locally convex space of all germs of analytic functions on $K$ with the usual inductive limit topology.

Given a compact set $K$ on an open set $D \subset \Omega$ the restriction operator $J=$ $J_{D, K}: A(D) \rightarrow A(K)$ is an operator which maps any function $f \in A(D)$ to the germ $\varphi=J f \in A(K)$, generated by $f$.

If $X, Y$ are locally convex spaces and $i: X \rightarrow Y$ is a linear continuous injection we say that $X$ is imbedded (linearly and continuously) into $Y$, identifying often $X$ with its image $i(X)$ and writing $X \hookrightarrow Y$. If this imbedding is dense (i.e. $i(X)$ is a dense set in $Y$ ) then the conjugate mapping $i^{*}:=Y^{*} \rightarrow X^{*}$ is also a linear continuous injection, so we can identify any linear functional $y^{*} \in Y^{*}$ with its image $y^{\prime}:=i^{*}\left(y^{*}\right)=\left.y^{*}\right|_{X}$ and write in this case that $Y^{*}=Y^{\prime}:=i^{*}\left(Y^{*}\right) \hookrightarrow X^{*}$; this imbedding is also dense if the space $X$ is reflexive.

In particular, for a pluriregular pair $(K, D)$ we shall write

$$
\begin{aligned}
A(D) & =J_{D, K}(A(D)) \hookrightarrow A(K) ; \\
A(K)^{*} & =A(K)^{\prime}:=J_{D, K}^{*}\left(A(K)^{*}\right) \hookrightarrow A(D)^{*} .
\end{aligned}
$$

Given an open set $D \subset \Omega$ the elements of the space $A(D)^{*}$ are called usually analytic functionals on $D$ (see, e.g., $[\mathbf{H r}]$ ); so, any functional from $A(K)^{*}$ is identified in (2.5) with the corresponding analytic functional on $D$. Given $F \subset D$ the nonbounded seminorm is introduced

$$
\left|x^{\prime}\right|_{F}^{*}:=\sup \left\{\left|x^{\prime}(x)\right|: x \in A(D),|x|_{F} \leq 1\right\}
$$

on $A(D)^{*}$, which is definitely a norm if $(K, D)$ with $K=\bar{F}$ is a pluriregular condenser.

Scales. A family of Banach spaces $X_{\alpha}, \alpha_{0} \leq \alpha \leq \alpha_{1}$, is called a scale of Banach spaces (or simply a scale) if for arbitrary $\alpha_{0} \leq \alpha<\beta \leq \alpha_{1}$ two conditions hold: 1 ) $X_{\beta} \hookrightarrow X_{\alpha}$ and 2) $\|x\|_{\gamma} \leq C(\alpha, \beta, \gamma)\left(\|x\|_{X_{\alpha}}\right)^{1-\tau(\gamma)}\left(\|x\|_{X_{\beta}}\right)^{\tau(\gamma)}$ with $\tau(\gamma)=\frac{\gamma-\alpha}{\beta-\alpha}$, $\alpha<\gamma<\beta$. In what follows we send the reader to the monograph [KPS] for further notions and results about scales.

Here we turn our attention to a particular case of Hilbert scale $H_{\alpha}=H_{0}^{1-\alpha} H_{1}^{\alpha}, \alpha \in$ $(-\infty, \infty)$, spanned on a couple of Hilbert spaces with a dense compact imbedding $H_{1} \hookrightarrow H_{0}$. Since under such assumptions there is a common orthogonal basis $\left\{\varphi_{i}\right\}$ for $H_{0}$ and $H_{1}$, normalized in $H_{0}$ and enumerated by non-decreasing of norms in the space $H_{1}$ :

$$
\left\|\varphi_{i}\right\|_{H_{0}}=1, i \in \mathbb{N} ; \mu_{i}=\mu_{i}\left(H_{0}, H_{1}\right):=\left\|\varphi_{i}\right\|_{H_{1}} \nearrow \infty,
$$

this scale is determined by the norms

$$
\|x\|_{H_{\alpha}}:=\left(\sum_{i \in \mathbb{N}}\left|\xi_{i}\right|^{2} \mu_{i}^{2 \alpha}\right)^{1 / 2}, x=\sum_{i \in \mathbb{N}} \xi_{i} \varphi_{i},
$$

so that the space $H_{\alpha}$ consists of $x \in H_{0}$ with a finite norm (2.8) if $\alpha \geq 0$, while $H_{\alpha}$ is the completion of $H_{0}$ by the norm (2.8) if $\alpha<0$. 
Diameters. We shall use the following equivalent definition of the Kolmogorov diameters (1.2):

$$
d_{i}\left(X_{1}, X_{0}\right)=\inf \left\{\inf \left\{\lambda>0: \mathbb{B}_{X_{1}} \subset \lambda \mathbb{B}_{X_{0}}+L\right\}: L \in \mathcal{L}_{i}\right\},
$$

where $\mathcal{L}_{i}$ is the set of all $i$-dimensional subspaces of $X_{0}$.

The following property follows directly from the definitions (1.2), (2.9).

Proposition 2.1. Let $X_{1} \hookrightarrow Y_{1} \hookrightarrow Y_{0} \hookrightarrow X_{0}$ be a quadruple of Banach spaces with dense imbeddings, then there is a constant $M$ such that

$$
d_{i}\left(X_{1}, X_{0}\right) \leq M d_{i}\left(Y_{1}, Y_{0}\right), \quad i \in \mathbb{N} .
$$

In the conditions concerned with (2.7) the following simple expression for the diameters holds (see, e.g., [M], Corollary 3) :

$$
d_{i}\left(H_{1}, H_{0}\right)=\frac{1}{\mu_{i+1}\left(H_{0}, H_{1}\right)}, \quad i \in \mathbb{N} .
$$

Hence for the Hilbert scale $H_{\alpha}=H_{0}^{1-\alpha} H_{1}^{\alpha}$, due to representation (2.8), we have the equality

$$
d_{i}\left(H_{\alpha_{1}}, H_{\alpha_{0}}\right)=\frac{1}{\mu_{i+1}\left(H_{0}, H_{1}\right)^{\alpha_{1}-\alpha_{0}}}=\left(d_{i}\left(H_{1}, H_{0}\right)\right)^{\alpha_{1}-\alpha_{0}}, \alpha_{0}<\alpha_{1} .
$$

\section{Hadamard type inequalities for analytic functions and functionals}

Analogously to the one-dimensional case, one of the main applications of Green pluripotential is Two Constants Theorem for analytic functions, in particular, Hadamard type interpolational estimates ([Si] , see also $[\mathbf{Z 3}]$ ), which may be written, for a pluriregular pair $(K, D)$, in the form

$$
|f|_{D_{\alpha}} \leq\left(|f|_{K}\right)^{1-\alpha}\left(|f|_{D}\right)^{\alpha}, 0<\alpha<1, f \in H^{\infty}(D),
$$

where the intermediate sets $D_{\alpha}$ are defined in (2.2). Those estimates are very useful for constructing of common bases for the spaces $A(K)$ and $A(D)([\mathbf{Z 1}, \mathbf{Z 3}, \mathbf{Z 6}$, $\mathbf{Z 7}$ ], see also $[\mathbf{N g}, \mathbf{Z S}, \mathbf{Z e 1}])$, since they provide good estimates for the system $\left\{f_{i}(z)\right\}$, examined for being a basis.

Hadamard type interpolational estimates for analytic functionals are of no less importance. They are needed to provide good estimates for the biorthogonal system of analytic functionals $\left\{f_{i}^{\prime}\right\}$. In the one-dimensional case, due to GrothendieckKöthe-Silva duality, analytic functionals can be represented as analytic functions in the complement of $K$, so one can use the same inequalities (3.1) to estimate functionals (see, e.g., $[\mathbf{Z 1}, \mathbf{N g}, \mathbf{Z S}]$ ). For $n \geq 2$, though this direct way fails, the following analogue of Two Constant Theorem for analytic functionals holds.

Theorem 3.1. ( $[\mathbf{Z 3}, \mathbf{Z 6}, \mathbf{Z 7}])$ Let $(K, D)$ be a pluriregular pair on a Stein manifold $\Omega$ and $D$ be strongly pluriregular. Then for each $\varepsilon>0$ and $\alpha \in(0,1)$ there is a constant $M=M(\alpha, \varepsilon)$ such that for any $x^{\prime} \in A C(K)^{\prime} \hookrightarrow A(D)^{*}$ the estimates hold:

$$
\left|x^{\prime}\right|_{D_{\alpha}}^{*} \leq M\left(\left|x^{\prime}\right|_{K}^{*}\right)^{1-\alpha+\varepsilon}\left(\left|x^{\prime}\right|_{D}^{*}\right)^{\alpha-\varepsilon} .
$$




\section{Adherent spaces}

Let $E$ be a Fréchet space, $\left\{\|x\|_{p}, p \in \mathbb{N}\right\}$ be a system of seminorms defining its topology,

$$
\left\|x^{*}\right\|_{p}^{*}:=\sup \left\{\left|x^{*}(x)\right|: x \in E,\|x\|_{p} \leq 1\right\}, \quad x^{*} \in E^{*}, p \in \mathbb{N},
$$

be the system of polar (non-bounded, in general) norms, and

$$
U_{p}:=\left\{x \in E:\|x\|_{p} \leq 1\right\}, \quad U_{p}^{\circ}:=\left\{x^{*} \in E^{*}:\left\|x^{*}\right\|_{p}^{*} \leq 1\right\}, \quad p \in \mathbb{N} .
$$

The following interpolation property proved to be useful in studying of structural properties of Fréchet spaces (see, e.g., [Z2, Z5, Vg, VW, MV]).

Definition 4.1. A Fréchet space $E$ satisfies the property $\mathcal{D}_{2}$ (we write also $E$ $\left.\in\left(\mathcal{D}_{2}\right)\right)$ if for every $p \in \mathbb{N}$ there is $q \in \mathbb{N}$ such that for each $r \in \mathbb{N}$ there is a constant $C$ providing the estimate:

$$
\left(\left\|x^{*}\right\|_{q}^{*}\right)^{2} \leq C\left\|x^{*}\right\|_{p}^{*}\left\|x^{*}\right\|_{r}^{*}, \quad x^{*} \in E^{*}
$$

Definition 4.2. A Banach space $X \hookrightarrow E$ is said to be adherent to $E$ if for each $p \in \mathbb{N}$ and any $\delta>0$ there is $q \in \mathbb{N}$ and a constant $C>0$ such that $\left\|x^{*}\right\|_{q}^{*} \leq C\left(\left\|x^{*}\right\|_{X^{*}}\right)^{1-\delta}\left(\left\|x^{*}\right\|_{p}^{*}\right)^{\delta}, \quad x^{*} \in E^{*}$.

Proposition 4.3. ([Vg, MV])A Banach space $X$ is adherent to $E$ if and only if for any neighborhood $V$ of zero in $E$ and each $\delta>0$ there is $p \in N$ and a constant $C>0$ such that

$$
U_{p} \subset t^{\delta} \mathbb{B}_{X}+\frac{C}{t^{1-\delta}} V, \quad t>0
$$

Proposition 4.4. (D. Vogt $[\mathbf{V g}]$, Lemma 4) Let a Schwartz Fréchet space E satisfy the property $\mathcal{D}_{2}$. Then there is a Banach space $X \hookrightarrow E$ adherent to $E$.

Proposition 4.5. Let $\Omega$ be a Stein manifold with a finite set of connected components. Then the following statements are equivalent: (i) $\Omega$ is pluriregular; (ii) $A(\Omega) \in \mathcal{D}_{2}$; (iii) there exists a Hilbert space $H \hookrightarrow A(\Omega)$ adherent to the space $A(\Omega)$.

Proof. The relations $(i) \Leftrightarrow($ ii $)$ and $($ iii $) \Rightarrow(i)$ are due to $[\mathbf{Z 3 ,} \mathbf{Z 5}]$ (see also, $[\mathbf{Z 6}, \mathbf{Z 7}])$, therewith the proof of $(i) \Rightarrow(i i)$ is based on Hadamard type inequalities for analytic functionals (see, Theorem 3.1 above); $(i i) \Leftrightarrow($ iii $)$ by Vogt's result (see, Proposition 4.4 above) there exists a Banach space $X \hookrightarrow A(\Omega)$ adherent to the space $A(\Omega)$ and, since $A(\Omega)$ is nuclear, then, due to Pietsch $[\mathbf{P i}]$, there exists a Hilbert space $H$ such that $X \hookrightarrow H \hookrightarrow A(\Omega)$. Hence $H$ is adherent to $A(\Omega)$.

It should be mentioned that Aytuna $[\mathbf{A}]$ constructed, under the assumption (i), an adherent Hilbert space for $A(\Omega)$ as a weighted $L_{2}$-space, applying Hörmander's $\bar{\partial}$-problem technics. For good enough domains we have the following easy description of adherent spaces for $A(\Omega)$ (see, e.g., $[\mathbf{Z 6}, \mathbf{Z 7}]$ ).

Proposition 4.6. Let $D$ be a strongly pluriregular domain on a Stein manifold. Then any Banach space $X$ satisfying the dense imbeddings $A(\bar{D}) \hookrightarrow X \hookrightarrow A(D)$ is adherent to $A(D)$; in particular, the space $H^{\infty}(D)$ is adherent to $A(D)$. 
Definition 4.7. Let $K$ be a compact set on a Stein manifold $\Omega$ and a Banach space $X$ be such that the dense imbedding $A(K) \hookrightarrow X$ holds. We say that $X$ is adherent to $A(K)$ if its dual $X^{*} \hookrightarrow A(K)^{*}$ is adherent to $A(K)^{*}$ (in the sense of Definition 4.2).

The following fact cannot be obtained from Proposition 4.5 for $\operatorname{dim} \Omega \geq 2$, because there is no proper multidimensional analogue of the Grothendieck-KötheSilva duality.

Proposition 4.8. ([Z3, Z4]) Let $K$ be a compact set on a Stein manifold $\Omega$ such that $A(\Omega)$ is dense in $A(K)$ (i.e. $K$ is a Runge set on $\Omega$ ) and $\Omega$ has no connected component disjoint with $K$. The following statements are equivalent: $(i)$ $K$ is pluriregular; (ii) $A(K)^{*} \in \mathcal{D}_{2}$; (iii) there is an adherent to $A(K)$ Hilbert space $H \hookleftarrow A(K)$; (iv) the space $A C(K)$ is adherent to $A(K)$.

It follows from $(i) \Rightarrow(i v)$ that any Hilbert space $H$, satisfying the dense imbeddings $A(K) \hookrightarrow H \hookrightarrow A C(K)$, is adherent to $A(K)$. A more explicit example of a Hilbert space adherent to $A(K)$ is the space $H=A L_{2}(K, \mu)$ obtained as a completion of $A(K)$ by the norm

$$
\|x\|:=\left(\int_{K}|x(z)|^{2} d \mu\right)^{1 / 2}
$$

where $\mu:=\left(d d^{c} \omega\right)^{n}$ with $\omega(z)=\omega(D, K ; z)$ and $D$ is any open set composing a pluriregular pair $(K, D)$ with $K([\mathbf{Z 6}, \mathbf{Z e 1}, \mathbf{Z} 7]$, see also $[\mathbf{Z e 2}]$ for a characterization of Borel measures $\mu$ providing that $H=A L_{2}(K, \mu)$ is adherent to $\left.A(K)\right)$.

Definition 4.9. Given a pluriregular pair $(K, \Omega)$ a couple of Banach spaces $\left(X_{0}, X_{1}\right)$ is said to be adherent to a couple $(A(K), A(\Omega))$ if

$$
X_{1} \hookrightarrow A(\Omega) \hookrightarrow A(K) \hookrightarrow X_{0}
$$

and $X_{1}$ is adherent to $A(\Omega), X_{0}$ is adherent to $A(K)$.

Propositions 4.5 and 4.8 yield the following

Corollary 4.10. For any pluriregular pair $(K, \Omega)$ there exists a couple of Banach (Hilbert) spaces $\left(X_{0}, X_{1}\right)$ adherent to $(A(K), A(\Omega))$.

The following statement was proved in $[\mathbf{Z 3}]$ for the particular case of Hilbert couples $\left(X_{0}, X_{1}\right)$; here we derive the general case from the Hilbert version using standard technics of Banach scales (see, e.g., [KPS]).

Theorem 4.11. Suppose that $(K, D)$ is a pluriregular pair, $D$ is a Stein manifold. Let $\left(X_{0}, X_{1}\right)$ be a couple of Banach spaces adherent to the couple $(A(K), A(D))$ such that $X_{1}$ is imbedded normally into $X_{0}$ and $\mathbb{B}_{X_{1}}$ is closed in $X_{0}$. Let $X_{\alpha}, 0 \leq \alpha \leq 1$, be any regular normal scale of Banach spaces connecting the spaces $X_{0}, X_{1}$. Then the following linear continuous imbeddings have place:

$$
A\left(K_{\alpha}\right) \hookrightarrow X_{\alpha} \hookrightarrow A\left(D_{\alpha}\right), \quad 0<\alpha<1,
$$

where $K_{\alpha}, D_{\alpha}$ have been defined in (2.2).

Proof. Consider any regular normal scale $X_{\alpha}, 0<\alpha<1$, connecting $X_{0}$ and $X_{1}$. First we are going to show that the imbeddings

$$
A(D) \hookrightarrow X_{\alpha} \hookrightarrow A(K)
$$


hold for each $\alpha \in(0,1)$. By the adherence of $X_{1}$ to $A(D)$, for each $\alpha<1$ there is $\beta<1$ such that

$$
\left|x^{\prime}\right|_{D_{\beta}}^{*} \leq C\left(\left\|x^{\prime}\right\|_{X_{0}^{*}}\right)^{1-\alpha}\left(\left\|x^{\prime}\right\|_{X_{1}^{*}}\right)^{\alpha}, x^{\prime} \in A(D)^{*} .
$$

Therefore for the minimal scale $X_{\alpha}^{\text {min }}$ connecting the spaces $X_{0}$ and $X_{1}$ ([KPS]) we obtain the estimate

$$
\|x\|_{X_{\alpha}^{\min }}:=\sup _{x^{\prime} \in X_{0}^{*}}\left\{\frac{\left|x^{\prime}(x)\right|}{\left(\left\|x^{\prime}\right\|_{X_{0}^{*}}\right)^{1-\alpha}\left(\left\|x^{\prime}\right\|_{X_{1}^{*}}\right)^{\alpha}}\right\} \leq C \sup _{x^{\prime} \in X_{0}^{*}}\left\{\frac{\left|x^{\prime}(x)\right|}{\left|x^{\prime}\right|_{D_{\beta}}^{*}}\right\}=C|x|_{D_{\beta}} .
$$

Hence $A(D) \hookrightarrow X_{\alpha}^{\min }, 0<\alpha<1$. Then, due to Lions-Peetre $[\mathbf{L P}]$ (see, also [KPS], Chapter IV, Theorem 2.20), the left imbedding in (4.7) holds for any scale $X_{\alpha}, 0<\alpha<1$, connecting $X_{0}$ and $X_{1}$.

The imbeddings (4.5) imply the natural dense imbeddings $X_{0}^{*} \hookleftarrow A(K)^{*} \hookleftarrow$ $A(D)^{*} \hookleftarrow Y_{0}$, where $Y_{0}$ is the closure of $A(\Omega)^{*}$ in $X_{1}^{*}$. Since $\mathbb{B}_{X_{1}}$ is closed in $X_{0}$ we have, by Aronszajn-Gagliardo [AG], that $Y_{0}$ is a norming set for $X_{1}$. Therefore, taking into account the reflexivity of $A(K)$, the adherence of $X_{1}$ to $A(K)$ implies that for any $\gamma>0$ there is $\delta>0$ and $C>0$ such that

$$
|x|_{D_{\delta}} \leq C\left(\|x\|_{X_{0}}\right)^{1-\gamma}\left(\|x\|_{X_{1}}\right)^{\gamma}, \quad x \in X_{1} .
$$

Then $\widehat{X}_{\gamma} \hookrightarrow A C\left(D_{\delta}\right) \hookrightarrow A(K)$, where $\widehat{X}_{\theta}:=\left(X_{0}, X_{1}\right)_{\theta, L_{1, *}, L_{1, *}}$ is the maximal scale of means ([KPS], Chapter IV, Lemma 2.6). Therefore, applying this imbedding with $\gamma<\alpha / 2$ and taking into account that any regular scale is almost imbedded into any scale ([KPS], Chapter IV, Corollary 3), we obtain the right imbedding in (4.6).

Now we take any pair of Hilbert spaces $H_{0}, H_{1}$ satisfying the imbeddings

$$
X_{1} \hookrightarrow H_{1} \hookrightarrow A(D) \hookrightarrow A(K) \hookrightarrow H_{0} \hookrightarrow X_{0} .
$$

Then by (4.7) the imbeddings

$$
X_{1} \hookrightarrow H_{1} \hookrightarrow X_{1-\varepsilon} \hookrightarrow X_{\varepsilon} \hookrightarrow H_{0} \hookrightarrow X_{0}
$$

hold for every $\varepsilon: 0<\varepsilon<1 / 2$. Applying now (4.7) to the Hilbert scale $H_{\alpha}=$ $\left(H_{0}\right)^{1-\alpha}\left(H_{1}\right)^{\alpha}$, which is true due to $[\mathbf{Z 3}, \mathbf{Z 6}, \mathbf{Z 7}]$, and using the interpolation property of scales $[\mathbf{K P S}]$, we obtain the imbeddings

$$
X_{\alpha+\varepsilon(1-\alpha)} \hookrightarrow H_{\alpha} \hookrightarrow A\left(D_{\alpha}\right), \quad A\left(K_{\alpha}\right) \hookrightarrow H_{\alpha} \hookrightarrow X_{\alpha(1-\varepsilon)}, 0<\alpha<1 .
$$

Since $\varepsilon>0$ may be taken arbitrarily small here, we obtain (4.6) that ends the proof.

The following result will be useful for investigating the problem about the asymptotics (1.7).

Theorem 4.12. Let a Hilbert space $X_{0} \hookleftarrow A(K)$ and a Banach space $X_{1} \hookrightarrow$ $A(\Omega)$ make a couple adherent to $(A(K), A(\Omega))$. Then the pair $\left(X_{0}, X_{1}\right)$ is admissible for $(K, \Omega)$.

PROOF. Let $Y_{0}, Y_{1}$ be a couple of Banach spaces satisfying the linear continuous imbeddings from Definition 1.3. We need to prove that

$$
\ln d_{i}\left(Y_{1}, Y_{0}\right) \sim \ln d_{i}\left(X_{1}, X_{0}\right) \text { as } i \rightarrow \infty .
$$


By Pietsch $[\mathbf{P i}]$, Proposition 4.4.1, there exists a Hilbert space $H_{1}$ satisfying the continuous imbeddings $X_{1} \hookrightarrow H_{1} \hookrightarrow A(\Omega)$, which is obviously adherent to $A(\Omega)$. Then, by Theorem 4.11, the Hilbert scale $H_{\alpha}=\left(X_{0}\right)^{1-\alpha}\left(H_{1}\right)^{\alpha}$ satisfies the continuous imbeddings (4.6). Then, by these imbeddings, the system of norms $\|x\|_{H_{\alpha}}, \alpha<$ 1 , defines the original topology of the space $A(\Omega)$. Since $X_{1}$ is adherent to the space $E=A(\Omega)$, we obtain that for arbitrary $\delta>0$ there exists $\alpha=\alpha(\delta)<1$ and $C=C(\delta)>0$ such that

$$
\mathbb{B}_{H_{\alpha}} \subset\left(\frac{1}{\lambda}\right)^{\delta} \mathbb{B}_{X_{1}}+C \lambda^{1-\delta} \mathbb{B}_{X_{0}}, \quad \lambda>0
$$

Now take an arbitrary $\lambda>0$ such that

$$
d_{i}\left(X_{1}, X_{0}\right)<\lambda<2 d_{i}\left(X_{1}, X_{0}\right) .
$$

By the definition, there is $L \in \mathcal{L}_{i}$ such that

$$
\mathbb{B}_{X_{1}} \subset \lambda \mathbb{B}_{X_{0}}+L
$$

Combining (4.9), (4.11), we obtain that

$$
\mathbb{B}_{H_{\alpha}} \subset(1+C) \lambda^{1-\delta} \mathbb{B}_{X_{0}}+L
$$

Then, due to (4.10), we obtain

$$
d_{i}\left(H_{\alpha}, X_{0}\right) \leq(1+C)\left(2 d_{i}\left(X_{1}, X_{0}\right)\right)^{(1-\delta)} .
$$

Let $\alpha>\beta>0$. Then, taking into account (2.12), we get from here that

$$
\begin{aligned}
d_{i}\left(X_{1}, X_{0}\right) & \prec d_{i}\left(Y_{1}, Y_{0}\right) \prec d_{i}\left(H_{\alpha}, H_{\beta}\right)=\left(d_{i}\left(H_{1}, X_{0}\right)\right)^{\frac{\alpha-\beta}{\alpha}} \\
& \prec\left(d_{i}\left(X_{1}, X_{0}\right)\right)^{\frac{(1-\delta)(\alpha-\beta)}{\alpha}},
\end{aligned}
$$

Since $\beta$ can be taken arbitrarily small, we obtain (4.8).

Corollary 4.13. Let a Banach space $X_{1} \hookrightarrow A(\Omega)$ be adherent to $A(\Omega)$. Then the couple $\left(A C(K), X_{1}\right)$ is admissible for $(K, \Omega)$.

Proof. Since the Hilbert space $X_{0}=A L^{2}(K, \mu)$ (see the paragraph preceding Definition 4.9) is adherent to $A(K)$ and $A(K) \hookrightarrow A C(K) \hookrightarrow X_{0}$, we obtain, applying Theorem 4.12 , that $\left(A C(K), X_{1}\right)$ is admissible for $(K, \Omega)$.

REMARK 4.14. The admissibility is, in general, essentially weaker than the adherence. Indeed, consider the simplest pair of two concentric disks $K=\overline{\mathbb{D}}$, $D=\mathbb{D}_{R}, \quad R>1$. Define the couple of Hilbert spaces $\left(H_{0}, H_{1}\right)$ by the norms

$$
\|x\|_{\nu}:=\left(\sum_{k=0}^{\infty}\left|\xi_{k}\right|^{2} a_{k}^{(\nu)}\right)^{1 / 2}, x(z)=\sum_{k=0}^{\infty} \xi_{k} z^{k}, \quad \nu=0,1
$$

with $a_{k}^{(\nu)}:=R^{-\nu k}$ if $k \neq 2^{j}$ and $a_{k}^{(\nu)}:=(2 R)^{-\nu k}$ if $k=2^{j}, j \in \mathbb{N}$. An easy calculation shows that $\ln d_{i}\left(H_{1}, H_{0}\right) \sim-\frac{i}{\ln R}=-\frac{i}{\tau(K, D)}$ as $i \rightarrow \infty$, hence the couple $\left(H_{0}, H_{1}\right)$ is admissible for $(K, D)$, but it is not adherent to $(A(K), A(D))$.

Problem 4.15. Let $\left(A C(K), H^{\infty}(D)\right)$ be an admissible couple for a pluriregular pair $(K, D)$. Is then $H^{\infty}(D)$ adherent to $A(D)$ ? 


\section{Maximal plurisubharmonic functions with isolated singularities}

Let $\Omega$ be a pluriregular Stein manifold. Given a finite set $\Lambda=\left\{\zeta_{\mu}: \mu=1, \ldots, m\right\} \subset$ $\Omega$, denote by $\mathfrak{G}_{\Lambda}(\Omega)$ the class of all functions $u \in P \operatorname{sh}(\Omega) \cap M P(\Omega \backslash \Lambda)$ taking the value $-\infty$ on $\Lambda$ and satisfying the conditions $\lim _{j \rightarrow \infty} u\left(z_{j}\right)=0$ for any sequence $\left\{z_{j}\right\}$ discrete in $\Omega$ (or, shortly, $\lim _{z \rightarrow \partial \Omega} u(z)=0$ ). In particular, if $\Omega$ is a regular one-dimensional Stein manifold, then every function $u \in \mathfrak{G}(\Omega)$ is represented in the form

$$
u(z)=-\sum_{\mu=1}^{m} \alpha_{\mu} G_{\Omega}\left(\zeta_{\mu}, z\right),
$$

where $\alpha_{\mu}>0$ and $G_{\Omega}(\zeta, z)$ is the Green function for $\Omega$ with the unit logarithmic singularity at the point $\zeta$. It is well-known how important are those functions in onedimensional complex analysis, especially in the approximation and interpolation theory.

By contrast, the multidimensional case is much more complicated: on the one hand, usually a function $u \in \mathfrak{G}(\Omega)$ with more than one pole is not representable as a sum of functions with single poles and, on the other hand, isolated singularities of such functions are extremely varied (as seen from the following example).

EXAMPLE 5.1. Let $\Delta$ be a complete logarithmically convex $n$-circular domain, relatively compact in a unit polydisc $\mathbb{U}^{n}$, and

$$
h(\theta)=h_{\Delta}(\theta):=\sup \left\{\sum_{\nu=1}^{n} \theta_{\nu} \ln \left|z_{\nu}\right|: z=\left(z_{\nu}\right) \in \Delta\right\}
$$

its characteristic function. Then every function

$$
k_{\Delta}(z):=\sup \left\{\frac{\sum_{\nu=1}^{n} \theta_{\nu} \ln \left|z_{\nu}\right|}{-h(\theta)}: \theta=\left(\theta_{\nu}\right) \in \Sigma\right\}, z \in \mathbb{U}^{n}
$$

is of the class $\mathfrak{G}_{\{0\}}\left(\mathbb{U}^{n}\right)$. All this singularities at the origin are different, in the sense of the following.

Definition 5.2. Given a point $\zeta \in \Omega$ we consider the set of all functions $\varphi \in \operatorname{Psh}(U) \cap M P(U \backslash\{\zeta\})$ with $\varphi(\zeta)=-\infty$, where $U=U(\varphi)$ is an open neighborhood of $\zeta$ and define the equivalence relation by

$$
\varphi \sim \psi \stackrel{\text { def }}{=} \lim _{z \rightarrow \zeta} \frac{\varphi(z)}{\psi(z)}=1 .
$$

Denote by $\mathfrak{S}_{\zeta}$ the set of all equivalence classes under the relation (5.3) (we call them also "standard singularities" at the point $\zeta$ ) and write $\sigma=[\varphi]$ if $\varphi \in \sigma \in \mathfrak{S}_{\zeta}$. A singularity $\sigma \in \mathfrak{S}_{\zeta}$ is called continuous if there is a representative $\varphi \in \sigma$, continuous in some punctured neighborhood of $\zeta$.

Theorem 5.3. ([Z6, Z7] ) Given a pluriregular Stein manifold $\Omega$, a finite set $F=\left\{\zeta_{\mu}: \mu=1, \ldots, m\right\}$ on it and continuous standard singularities $\sigma_{\mu}=\left[\varphi_{\mu}\right] \in$ $\mathfrak{S}_{\zeta_{\mu}}, \mu=1, \ldots, m$, there exists the unique function $g \in \mathfrak{G}_{F}(\Omega)$ having the singularities $\sigma_{\mu}$ at the points $\zeta_{\mu}$. This function is continuous in $\Omega \backslash F$ and is defined by the formula:

$$
g(z)=g_{\Omega}\left(F,\left(\sigma_{\mu}\right) ; z\right):=\sup \left\{u(z): u \in P\left(\Omega, F,\left(\sigma_{\mu}\right)\right)\right\},
$$


where $P\left(\Omega, F,\left(\sigma_{\mu}\right)\right)$ is the class of all functions $u \in P$ sh $(\Omega)$ such that $u(z)<0$ in $\Omega$ and there is a constant $c=c(u)$ provided the estimate $u(z) \leq \varphi_{\mu}(z)+c$ in some neighborhood of $\zeta_{\mu}, \mu=1, \ldots, m$.

Proof. We suppose, without loss of generality, that the neighborhoods $U_{\mu}=$ $U\left(\varphi_{\mu}\right)$ are disjoint, set $U:=\cup U_{\mu}$ and define a function $\varphi$ on $U$ so that $\varphi(z):=$ $\varphi_{\mu}(z)$ for $z \in U_{\mu}$. Consider the sets

$$
\Phi_{\lambda}=\{z \in U: \varphi(z)<-\lambda\} ; F_{\lambda}=\{z \in U: \varphi(z) \leq-\lambda\}, 0<\lambda<\infty .
$$

Choose $\delta>0$ such that $F_{\delta}$ is compact in $\Omega$. For any $u \in P\left(\Omega, F,\left(\sigma_{\mu}\right)\right)$ find constants $c$ and $\tau \geq \delta$ such that $u(z) \leq \varphi(z)+c$ if $z \in F_{\tau}$. Take $\lambda_{0}=\max \{c, \tau\}$. The function $\psi(z)=\frac{\lambda-c}{\lambda-\delta}(\varphi(z)+\delta)$ is maximal plurisubharmonic function in $U$ for $\lambda>\lambda_{0}$ and, by the construction, $u(z) \leq \psi(z)$ if $z \in \partial\left(\Phi_{\delta} \backslash F_{\lambda}\right)$. Hence,

$$
u(z) \leq \frac{\lambda-c}{\lambda-\delta}(\varphi(z)+\delta), z \in \Phi_{\delta} \backslash F_{\lambda}, \lambda>\lambda_{0} .
$$

Tending $\lambda$ to infinity in this inequality, we obtain the estimate $u(z) \leq \varphi(z)+\delta$ in $\Phi_{\delta}$ for any $u \in P\left(\Omega, \Lambda,\left(\sigma_{\mu}\right)\right)$ and derive from here that the function $\widetilde{g}:=\limsup _{\zeta \rightarrow z} g(\varsigma)$ satisfies the estimate

$$
\widetilde{g}(z) \leq \varphi(z)+\delta, z \in \Phi_{\delta},
$$

hence belongs to the class $P\left(\Omega, F,\left(\sigma_{\mu}\right)\right)$, therefore $g(z) \equiv \widetilde{g}(z)$. Then the function

$$
v(z):=\left\{\begin{array}{cc}
g(z), & z \in \Omega \backslash \Phi_{\delta} \\
\max \{g(z), \varphi(z)+\delta-\gamma\}, & z \in \Phi_{\delta}
\end{array},\right.
$$

where $\gamma:=-\inf \left\{g(z): z \in \partial \Phi_{\delta}\right\}$, belongs to the class $P\left(\Omega, F,\left(\sigma_{\mu}\right)\right)$ itself, hence $v(z) \leq g(z)$. Therefore

$$
\varphi(z) \leq g(z)-\delta+\gamma, z \in \Phi_{\delta} .
$$

From $g(z)=\widetilde{g}(z) \leq 0$ and (5.5), (5.6) we derive that

$$
g(z) \leq(\lambda+\delta)\left(\omega\left(\Omega, F_{\lambda} ; z\right)-1\right), z \in \Omega \backslash F_{\lambda}, \lambda>\lambda_{0} .
$$

On the other hand, due to (5.7), if $\lambda>\lambda_{1}:=\max \left\{\gamma, \lambda_{0}\right\}$, the function

$$
w(z):=\left\{\begin{array}{cc}
\max \left\{g(z),(\lambda+\delta-\gamma)\left(\left(\omega\left(\Omega, F_{\lambda} ; z\right)-1\right)\right)\right\}, & z \in \Omega \backslash F_{\lambda} \\
g(z), & z \in F_{\lambda}
\end{array}\right.
$$

belongs to the class $P\left(\Omega, F,\left(\sigma_{\mu}\right)\right)$, which provides, together with (5.8), the estimates

$$
(\lambda+\delta-\gamma)\left(\left(\omega\left(\Omega, F_{\lambda} ; z\right)-1\right)\right) \leq g(z) \leq(\lambda+\delta)\left(\omega\left(\Omega, F_{\lambda} ; z\right)-1\right)
$$

for $z \in \Omega \backslash F_{\lambda}, \lambda>\lambda_{1}$. We conclude from here that

$$
g(z)=\lim _{\lambda \rightarrow \infty} \lambda\left(\omega\left(\Omega, F_{\lambda} ; z\right)-1\right),
$$

with uniform convergence on any compact subset of $\Omega \backslash F$. Since the pair $\left(\Omega, F_{\lambda}\right)$ is pluriregular for each $\lambda$, the function $\lambda\left(\omega\left(\Omega, F_{\lambda} ; z\right)-1\right)$ is continuous in $\Omega$ for each $\lambda$, hence the function $g$ is continuous in $\Omega \backslash F$. From (5.9) we derive also that $\lim _{z \rightarrow \partial \Omega} g(z)=0$, so $g \in \mathfrak{G}_{F}(\Omega)$. 
Corollary 5.4. Given a finite set $F=\left\{\zeta_{\mu}: \mu=1, \ldots, m\right\}$ on a pluriregular Stein manifold and a vector $\alpha=\left(\alpha_{\mu}\right) \in \mathbb{R}_{+}^{m}$ there is the unique function $g(z)=$ $g_{\Omega}(F, \alpha ; z) \in \mathfrak{G}_{F}(\Omega)$ having at the point $\zeta_{\mu}$ the standard singularity $\sigma_{\mu}=\left[\varphi_{\mu}\right]$ defined by the function $\varphi_{\mu}(z):=\alpha_{\mu} \ln \left|t_{\mu}\left(\zeta_{\mu}\right)-t_{\mu}(z)\right|$, where $t_{\mu}: U_{\mu} \rightarrow \mathbb{C}^{n}$ are local coordinates in a neighborhood $U_{\mu}$ of the point $\zeta_{\mu}, \mu=1, \ldots, m$ (we call this function multipolar Green function).

Definition 5.5. ([Z6, Z7]; cf.,[BT2], section 9) Let $u \in P \operatorname{sh}(\Omega), E \Subset \Omega$. The MP-balayage (sweeping out) of the function $u$ with respect to the set $\Omega \backslash E$ is the function

$$
s(z)=s(E, u ; z):=\limsup _{\zeta \rightarrow z} \sup \{v(\zeta): v \in P(\Omega, E ; u)\},
$$

where $P(\Omega, E ; u)$ is the class of all functions $v \in P \operatorname{sh}(\Omega)$, satisfying $v(z) \leq$ $u(z), z \in \Omega \backslash E$.

Proposition 5.6. Let $u \in C(\Omega) \cap P \operatorname{sh}(\Omega), G \Subset D \Subset \Omega, G$ strictly regular open set. Then

$$
\int_{D}\left(d d^{c} u(z)\right)^{n}=\int_{D}\left(d d^{c} s(G, u ; z)\right)^{n} .
$$

Proof. Let first $u \in C^{2}\left(\Omega^{\prime}\right), D \Subset \Omega^{\prime} \Subset \Omega, \partial D \in C^{1}$. Then, by Stokes' formula,

$$
\int_{D}\left(d d^{c} u\right)^{n}=\int_{\partial D} d^{c} u \wedge\left(d d^{c} u\right)^{n-1} .
$$

It is easily seen that Stokes' formula can be applied to functions which are $C^{2}$ only in a neighborhood of the boundary $\partial D$ and so, to the function $s(z):=s(G, u ; z)$. Since $u(z) \equiv s(z)$ in $\Omega \backslash G$, we obtain (5.11) and, furthermore, the smoothness assumption on $\partial D$ can be dropped.

In the general case, take a sequence $u_{\nu} \in C^{2}\left(\overline{\Omega^{\prime}}\right) \cap P \operatorname{sh}\left(\Omega^{\prime}\right), D \Subset \Omega^{\prime} \Subset \Omega$, such that $\delta_{\nu}:=\left|u-u_{\nu}\right|_{\overline{\Omega^{\prime}}} \rightarrow 0$. Let $s_{\nu}(z):=s\left(G, u_{\nu} ; z\right)$. Since $u_{\nu}(z) \equiv s_{\nu}(z)$ in $\Omega^{\prime} \backslash G$ and $s$ and $s_{\nu}$ are maximal in $G$, we obtain that

$$
s(z)-\delta_{\nu} \leq s_{\nu}(z) \leq s(z)+\delta_{\nu}, \quad z \in \bar{G} .
$$

Therefore $\left|s-s_{\nu}\right|_{\overline{\Omega^{\prime}}} \rightarrow 0$ as $\nu \rightarrow 0$. By continuity of the Monge-Ampére operator with respect to the compact convergence (see, e.g., [CLN, BT1, Sa1]), the limit transition in the formula (5.11), with $u_{\nu}, s_{\nu}$ instead of $u, s$, gives this formula in general case.

DeFinition 5.7. Given a point $\zeta$ on a Stein manifold $\Omega, \operatorname{dim} \Omega=n$, and $\sigma=[\varphi] \in \mathfrak{S}_{\zeta}$ the charge of the standard singularity $\sigma$ is the value

$$
\nu_{\zeta}(\sigma)=\nu_{\zeta}\{\varphi\}:=\left(\frac{1}{2 \pi}\right)^{n} \int_{\Omega}\left(d d^{c} s\left(\Delta_{\lambda}, \varphi ; z\right)\right)^{n}, \lambda>\delta,
$$

where $\Delta_{\lambda}:=\{z \in \Delta: \varphi(z)<-\lambda\}, \Delta=\Delta(\varphi) \Subset \Omega$ is an open neighborhood of $\zeta$ provided $\varphi \in P \operatorname{sh}(\Delta) \cap M P(\Delta \backslash\{\zeta\})$ and $\delta=\delta(\varphi)$ is such that $\Delta_{\delta} \Subset \Omega$. 
It is clear that

$$
\omega\left(\Delta_{\lambda_{1}}, \overline{\Delta_{\lambda_{0}}} ; z\right)=\frac{s\left(\Delta_{\lambda_{0}}, \varphi ; z\right)+\lambda_{0}}{\lambda_{0}-\lambda_{1}}
$$

and

$$
\nu_{\zeta}\{\varphi\}=\left(\lambda_{0}-\lambda_{1}\right)^{n} C\left(\overline{\Delta_{\lambda_{0}}}, \Delta_{\lambda_{1}}\right),
$$

where $\delta<\lambda_{1}<\lambda_{0}$.

Proposition 5.8. The charge of a standard singularity is well-defined, i.e. the value (5.13) does not depend on a choice of $\lambda$ or $\varphi$.

Proof. The value (5.13) does not depend on $\lambda>\delta$, due to Proposition 5.6. To show that it is also independent of a choice of a representative $\varphi$ in the class $\sigma$, we take another representative $\varphi^{\prime} \in \sigma=[\varphi]$ Then for each $\varepsilon>0$ there is $\gamma=\gamma(\varepsilon)$ such that

$$
\Delta_{(1+\varepsilon) \lambda} \subset \Delta_{\lambda}^{\prime} \subset \Delta_{(1-\varepsilon) \lambda}, \lambda \geq \gamma,
$$

where $\Delta_{\lambda}^{\prime}$ are sublevel domains for the function $\varphi^{\prime}$. Using monotonicity of the capacity ([Sa1, BT2] ) and the relations (5.12), (5.14), (5.15) with $\lambda_{1}=\lambda>\gamma$ and $\lambda_{0}=2 \lambda$, we get

$$
\frac{\nu_{\zeta}\{\varphi\}}{(1+3 \varepsilon) \lambda} \leq \frac{\nu_{\zeta}\left\{\varphi^{\prime}\right\}}{\lambda} \leq \frac{\nu_{\zeta}\{\varphi\}}{(1-3 \varepsilon) \lambda}, \lambda \geq \gamma
$$

which implies the equality $\nu_{\zeta}\{\varphi\}=\nu_{\zeta}\left\{\varphi^{\prime}\right\}$, since $\varepsilon>0$ is arbitrary.

Definition 5.9. Given a function $g \in \mathfrak{G}(\Omega)$ with the set of singularities $F=$ $F(g)=\left\{\zeta_{\mu}: \mu=1, \ldots, m\right\}$ consider its sublevel domains

$$
\Omega_{\lambda}:=\{z \in \Omega: g(z)<-\lambda\}, 0<\lambda<\infty .
$$

The charge of $g$ (supported by the set $F$ ) is defined as the value

$$
\nu\{g\}:=\left(\frac{1}{2 \pi}\right)^{n} \int_{\Omega}\left(d d^{c} s\left(\Omega_{\lambda}, g ; z\right)\right)^{n}=\sum_{\mu=1}^{m} \nu_{\zeta_{\mu}}[g], \lambda>0 .
$$

Proposition 5.10. The charge of the multipole Green plurifunction $g(z)=g_{\Omega}(F, \alpha ; z)$ is the value

$$
\nu\{g\}=\frac{\lambda^{n} C\left(F_{\lambda}, \Omega\right)}{(2 \pi)^{n}}=\sum_{\mu=1}^{m}\left(\alpha_{\mu}\right)^{n}
$$

where $F_{\lambda}=\{z \in \Omega: g(z) \leq-\lambda\}, 0<\lambda<\infty$.

Proof. Proposition 5.6 gives that the charge $\nu(g)$ does not depend on the choice of local coordinates in the definition of the function $g_{\Omega}(F, \alpha ; z)$. Therefore, applying (5.13), (5.17) and the well-known Jensen equality (see, e.g.,[Kl], Example 6.5.6):

$$
\left(\frac{1}{2 \pi}\right)^{n} \int_{\partial \mathbb{B}(o, r)} d^{c}(\ln |z|) \wedge\left(d d^{c}(\ln |z|)\right)^{n-1}=1,
$$

we obtain (5.18). 


\section{Generalized Schwarz and Bernstein Lemmas}

Let $\Omega$ be a pluriregular Stein manifold, $\operatorname{dim} \Omega=n, F=\left\{\zeta_{\mu}: \mu=1, \ldots, m\right\} \subset$ $\Omega ; \sigma=\left(s_{\mu}\right) \in \mathbb{Z}_{+}^{m}$. Denote by $A_{0}((F, \sigma), \Omega)$ the ideal consisted of all functions $f \in$ $A(\Omega)$ vanishing on $F$ and having zero of order $\leq s_{\mu}$ at the point $\zeta_{\mu}, \mu=1, \ldots, m$, and set

$$
A_{0}^{\perp}(F, \sigma):=\left\{f^{\prime} \in A(F)^{\prime}: f^{\prime}(f)=0 \text { for all } f \in A_{0}((F, \sigma), \Omega)\right\}
$$

An analytic functional $f^{\prime} \in A_{0}^{\perp}(F, \sigma) \subset A(\Omega)^{\prime}$ is called a discrete rational functional having the poles of order at least $s_{\mu}$ at the point $\zeta_{\mu}, \mu=1, \ldots, m$.

The following statement may be considered as a generalization of the classical Schwarz Lemma.

Theorem 6.1. Let $\Omega$ be a pluriregular Stein manifold, $\operatorname{dim} \Omega=n$, and

$$
F=\left\{\zeta_{\mu}: \mu=1, \ldots, m\right\} \subset \Omega ; \sigma=\left(s_{\mu}\right) \in \mathbb{Z}_{+}^{m} .
$$

Let $f \in A_{0}((F, \sigma), \Omega)$. Then the estimates

$$
|f(z)| \leq|f|_{\Omega} \exp \left(s(\alpha) g_{\Omega}(F, \alpha ; z)\right), z \in \Omega,
$$

hold with $s(\alpha):=\inf \left\{\frac{s_{\mu}}{\alpha_{\mu}}: \mu=1, \ldots, m\right\}$.

Proof. It makes sense to consider (6.2) only for bounded functions $f$. The function $u(z):=\frac{\ln |f(z)|-\ln |f|_{\Omega}}{s(\alpha)}$ belongs to the class $P\left(\Omega, F,\left(\sigma_{\mu}\right)\right)$ with the singularities $\sigma_{\mu}$ defined by the function $\varphi_{\mu}(z):=\alpha_{\mu} \ln \left|t\left(\zeta_{\mu}\right)-t(z)\right|$, where $t$ : $U_{\mu} \rightarrow \mathbb{C}^{n}$ are a local coordinates in a neighborhood $U_{\mu}$ of the point $\zeta_{\mu}, \mu=1, \ldots, m$ . Therefore $u(z) \leq g_{\Omega}(F, \alpha ; z)$ in $\Omega$ which is equivalent to (6.2) .

Now we consider the statement which is a generalization of the classical BernsteinWalsh-Siciak Lemma (see, e.g., [Si, Z4]) in order to fit estimates of discrete rational functionals.

Theorem 6.2. Let $D$ be a strictly pluriregular open set on a Stein manifold $\Omega$, $F=\left\{\zeta_{\mu}: \mu=1, \ldots, m\right\} \subset D ; \sigma=\left(s_{\mu}\right) \in \mathbb{Z}_{+}^{m}$ and

$$
\Phi_{\lambda}=\Phi_{\lambda}(F, \alpha):=\left\{z \in D: g_{D}(F, \alpha ; z)<-\lambda\right\}, 0<\lambda<\infty .
$$

Suppose $f^{\prime} \in A_{0}^{\perp}(F, \sigma)$. Then for each $\delta>0$ the estimates

$$
\left|f^{\prime}\right|_{\Phi_{\lambda}}^{*} \leq C\left|f^{\prime}\right|_{D}^{*} \exp (\lambda+\delta) s, 0<\lambda<\infty,
$$

hold with some constant $C=C(\lambda, \delta)$ and $s=s(\alpha):=\sup \left\{\frac{s_{\mu}}{\alpha_{\mu}}: \mu=1, \ldots, m\right\}$.

Proof. For each point $\zeta_{\mu} \in F$ we choose some local coordinates

$$
t^{(\mu)}=\left(t_{j}^{(\mu)}\right): \Delta^{(\mu)} \rightarrow \mathbb{U}^{n},
$$

with mutually disjoint neighborhoods $\Delta^{(\mu)}$ and set $\Delta=\cup_{\mu=1}^{m} \Delta^{(\mu)}$. Then

$$
g_{\Delta}(F, \alpha ; z)=\alpha_{\mu} \max \left\{\ln \left|t_{j}^{(\mu)}(z)\right|: j=1, \ldots, n\right\}, z \in \Delta^{(\mu)}, \mu=1, \ldots, m .
$$

and

$$
\Delta_{\tau}:=\left\{z \in \Delta: g_{\Delta}(F, \alpha ; z)<-\tau\right\}=\cup_{\mu=1}^{m} \Delta_{\tau}^{(\mu)}
$$


where $\Delta_{\tau}^{(\mu)}=\left\{z \in \Delta^{(\mu)}:\left|t_{j}^{(\mu)}(z)\right|<\exp \left(-\frac{\tau}{\alpha_{\mu}}\right)\right\}, 0<\tau<\infty$. The system

$$
f_{k, \mu}(z):=\left\{\begin{array}{ll}
t^{(\mu)}(z)^{k}:=t_{1}^{(\mu)}(z)^{k_{1}} \cdots t_{n}^{(\mu)}(z)^{k_{n}}, & z \in \Delta^{(\mu)} \\
0, & z \in \Delta \backslash \Delta^{(\mu)}
\end{array},\right.
$$

with $k=\left(k_{1}, \ldots, k_{j}, \ldots, k_{n}\right) \in \mathbb{Z}_{+}^{n}$ and $\mu=1, \ldots, m$, forms a common basis for all the spaces $A\left(\Delta_{\tau}\right)$ and the space $A(F)$. Its biorthogonal system $\left\{f_{k, \mu}^{\prime}\right\} \subset A(F)^{\prime} \subset$ $A\left(\Delta_{\tau}\right)^{\prime}$ may be expressed by the formula

$$
f_{k, \mu}^{\prime}(f)=\left(\frac{1}{2 \pi i}\right)^{n} \int_{S} \frac{f\left(v_{\mu}(t)\right) d t}{t^{k+I}}, f \in A\left(\Delta_{\tau}\right), 0<\tau<\infty,
$$

where $v_{\mu}: \mathbb{U}^{n} \rightarrow \Delta_{\mu}$ is the mapping inverse to the coordinate mapping (6.5), $\mu=1, \ldots, m, k=\left(k_{1}, \ldots, k_{j}, \ldots, k_{n}\right) \in \mathbb{Z}_{+}^{n}, I=(1, \ldots, 1)$ and $S=S_{\tau, \mu}$ is the Shilov boundary of the polydisc $\mathbb{U}_{r}^{n}$, where $r=r(\lambda):=\exp \left(-\lambda-\tau / \alpha_{\mu}\right)$, $0<\lambda<\infty$. It is easily seen that

$$
\left|f_{k, \mu}\right|_{\Delta_{\tau}}=\exp \left(-\frac{\tau|k|}{\alpha_{\mu}}\right) ;\left|f_{k, \mu}^{\prime}\right|_{\Delta_{\tau}}^{*}=\exp \left(\frac{\tau|k|}{\alpha_{\mu}}\right), 0<\tau<\infty .
$$

Since any functional $f^{\prime} \in A_{0}^{\perp}(F, \sigma)$ is represented in the form

$$
f^{\prime}=\sum_{\mu=1}^{m} \sum_{|k| \leq s_{\mu}} f^{\prime}\left(f_{k, \mu}\right) f_{k, \mu}^{\prime},
$$

using (6.9), we obtain the estimate:

$$
\begin{aligned}
\left|f^{\prime}\right|_{\Delta_{\tau}}^{*} & \leq\left|f^{\prime}\right|_{\Delta_{\tau}}^{*} \sum_{\mu=1}^{m}\left(\alpha_{\mu}\right)^{n}\left(\frac{s_{\mu}}{\alpha_{\mu}}\right)^{n} \exp \frac{\tau s_{\mu}}{\alpha_{\mu}} \\
& \leq\left|f^{\prime}\right|_{\Delta_{\tau}}^{*} \sum_{\mu=1}^{m}\left(\alpha_{\mu}\right)^{n} s(\alpha)^{n} \exp \tau s(\alpha)
\end{aligned}
$$

with $0<\tau<\infty$. So, for each $\varepsilon<0$ the estimates

$$
\left|f^{\prime}\right|_{\Delta_{\tau}}^{*} \leq L(\varepsilon)\left|f^{\prime}\right|_{\Delta}^{*} \exp (\tau+\varepsilon) s, 0<\tau<\infty,
$$

hold with $L(\varepsilon)=\sum_{\mu=1}^{m}\left(\alpha_{\mu}\right)^{n} \sup \left\{x^{n} \exp (-\varepsilon x): x>0\right\}$, where $s=s(\alpha):=\sup \left\{\frac{s_{\mu}}{\alpha_{\mu}}: \mu=1, \ldots, m\right\}$.

Choose $\gamma>0$ so that $\Phi_{\gamma} \Subset \Delta$. Since $\Delta_{\tau} \subset \Phi_{\tau}, 0<\tau<\infty$, we first derive from (6.10) a quite rough estimate for the left-hand side of (6.4):

$$
\left|f^{\prime}\right|_{\Phi_{\tau}}^{*} \leq L(\varepsilon)\left|f^{\prime}\right|_{\Phi_{\gamma}}^{*} \exp (\tau+\varepsilon) s, 0<\tau<\infty .
$$

Using the notation $F_{\tau}:=\left\{z \in D: g_{D}(F, \alpha ; z) \leq-\tau\right\}$, we have the relation:

$$
\omega\left(D, F_{\tau} ; z\right)=\frac{1}{\tau} g_{D}(F, \alpha ; z)+1, z \in D \backslash F_{\tau}, 0<\tau<\infty,
$$

which implies that

$$
D_{\alpha}^{(\tau)}:=\left\{z \in D: \omega\left(D, F_{\tau} ; z\right)<\alpha\right\}=\Phi_{(1-\alpha) \tau}, 0<\alpha<1, \quad 0<\tau<\infty .
$$

Therefore, applying (3.2) with $K=F_{\tau}, \alpha=1-\lambda / \tau, 0<\lambda<\tau, \varepsilon<0$, we obtain the estimate

$$
\left|f^{\prime}\right|_{\Phi_{\lambda}}^{*} \leq M\left(\left|f^{\prime}\right|_{F_{\tau}}^{*}\right)^{\lambda / \tau+\varepsilon}\left(\left|f^{\prime}\right|_{D}^{*}\right)^{1-\lambda / \tau-\varepsilon}
$$


with some constant $M=M(\tau, \lambda, \varepsilon)$.

Since the relation (6.4) is homogeneous, it is sufficient to prove it in the case when $\left|f^{\prime}\right|_{D}^{*}=1$. Under this assumption, taking into account (6.11) and (6.13), we obtain the estimate:

$$
\left|f^{\prime}\right|_{\Phi_{\lambda}}^{*} \leq M\left(L(\varepsilon)\left|f^{\prime}\right|_{\Phi_{\gamma}}^{*} \exp (\tau+\varepsilon) s\right)^{\frac{\lambda}{\tau}+\varepsilon} \leq M^{\prime}\left(\left|f^{\prime}\right|_{\Phi_{\gamma}}^{*}\right)^{\lambda / \tau+\varepsilon} \exp \left(\lambda+\varepsilon^{\prime}\right) s
$$

with some constant $M^{\prime}=M^{\prime}(\tau, \lambda, \varepsilon)$ and $\varepsilon^{\prime}=\tau \varepsilon+\varepsilon+\varepsilon^{2}, 0<\lambda<\tau$. Applying this estimate for $\lambda=\gamma$ with $\tau=4 \gamma$ and $\varepsilon=1 / 4$ we obtain that

$$
\left|f^{\prime}\right|_{\Phi_{\gamma}}^{*} \leq\left(M^{\prime}\right)^{2} \exp 2(2 \gamma+1) s .
$$

Given $\delta>0$ and $\lambda>0$ we choose $\tau=\tau(\lambda, \delta)$ and $\varepsilon=\varepsilon(\lambda, \delta)$ so that

$$
2(2 \gamma+1)(\lambda / \tau+\varepsilon)<\delta / 2 ; \tau \varepsilon+\varepsilon+\varepsilon^{2}<\delta / 2 .
$$

Then putting (6.15) into (6.14) considered with parameters satisfying the conditions (6.16) we obtain the estimate (6.4) in the case $\left|f^{\prime}\right|_{D}^{*}=1$ with some constant $C=$ $(\lambda, \delta)$. This completes the proof.

Corollary 6.3. Let $\Omega$ be a pluriregular Stein manifold of dimension $n$ and $F=\left\{\zeta_{\mu}: \mu=1, \ldots, m\right\} \subset \Omega ; \sigma=\left(s_{\mu}\right) \in \mathbb{Z}_{+}^{m}$. Let $H \hookrightarrow A(\Omega)$ be a Hilbert space adherent to $A(\Omega)$ and the dual space $A(\Omega)^{*}$ is considered as imbedded naturally into $H^{*}$. Then for each $f^{\prime} \in A_{0}^{\perp}(F, \sigma)$ and for any $\delta>0$ the estimates

$$
\left|f^{\prime}\right|_{\Omega_{\lambda}}^{*} \leq C\left\|f^{\prime}\right\|_{H^{*}} \exp (\lambda+\delta) s, 0<\lambda<\infty,
$$

hold with some constant $C=C(\lambda, \delta)$ and $s=s(\alpha):=\sup \left\{\frac{s_{\mu}}{\alpha_{\mu}}: \mu=1, \ldots, m\right\}$.

\section{Extendible bases}

Theorem 7.1. ([Z66, Z7] ) Let $F=\left\{\zeta_{\mu}: \mu=1, \ldots, m\right\}$ be a finite set on a pluriregular Stein manifold, $\operatorname{dim} \Omega=n$, having no connected component disjoint with the set $F ; \alpha=\left(\alpha_{\mu}\right)_{\mu=1}^{m}, \alpha_{\mu}>0, \mu=1, \ldots, m$. Let $g_{\Omega}(F, \alpha ; z)$ be the corresponding Green multipole function,

$$
\Omega_{\lambda}:=\left\{z \in \Omega: g_{\Omega}(F, \alpha ; z)<-\lambda\right\}, F_{\lambda}:=\left\{z \in \Omega: g_{\Omega}(F, \alpha ; z) \leq-\lambda\right\},
$$

with $0<\lambda<\infty$, and

$$
\sigma_{n}=\left(\frac{n !}{\sum_{\mu=1}^{m}\left(\alpha_{\mu}\right)^{n}}\right)^{1 / n} .
$$

Then there exists a common basis $\left\{f_{i}(z)\right\}_{i \in \mathbb{N}}$ for all the spaces

$$
A(\Omega), A(F), A\left(\Omega_{\lambda}\right), A\left(F_{\lambda}\right), 0<\lambda<\infty,
$$

such that for each $\varepsilon>0$ and $0<\lambda<\infty$ the estimates

$$
\frac{1}{C} \exp \sigma_{n}(-\lambda-\varepsilon) i^{1 / n} \leq\left|f_{i}(z)\right|_{F_{\lambda}} \leq C \exp \sigma_{n}(-\lambda+\varepsilon) i^{1 / n}, i \in \mathbb{N},
$$

hold with some constant $C=C(\lambda, \varepsilon)$. The Green multipole function can be expressed via the basis by the formula:

$$
\sigma_{n} g_{\Omega}(F, \alpha ; z)=\limsup _{\zeta \rightarrow z} \limsup _{i \rightarrow \infty} \frac{\ln \left|f_{i}(\zeta)\right|}{i^{1 / n}}, z \in \Omega \backslash F .
$$


Proof. Consider a system of analytic functionals

$$
\left\{f_{k, \mu}^{\prime}, k \in \mathbb{Z}_{+}^{n}, \mu=1, \ldots, m\right\} \subset A(F)^{\prime} \subset A(\Omega)^{\prime},
$$

biorthogonal to the basis in $A(F)$ consisted of monomials in local coordinates (see the beginning part of the proof of Theorem 6.2). Enumerate it into the sequence

$$
e_{i}^{\prime}=f_{k(i), \mu(i)}^{\prime}, i \in \mathbb{N},
$$

so that the sequence $s_{\alpha}(i):=\frac{|k(i)|}{\alpha_{\mu(i)}}$ will be non-decreasing.

Taking any Hilbert space $H$ adherent to $A(\Omega)$, we consider the dual space $A(\Omega)^{*}$ as imbedded naturally into $H^{*}$ (see, Preliminaries, Spaces). The system (7.6) is linearly independent and complete in $H^{*}$. Orthonormalizing it in the space $H^{*}$, we obtain the system of analytic functionals ("polynomials with respect to the system (7.6)"):

$$
\varphi_{i}^{\prime}=\sum_{j \leq i} b_{i j} e_{j}^{\prime}, i \in \mathbb{N}
$$

belonging to $A(F)^{\prime} \subset A(\Omega)^{*} \subset H^{*}$.

We are going to show that the system $\left\{\varphi_{i}\right\}_{i \in \mathbb{N}} \subset H \subset A(\Omega) \subset A(F)$, biorthogonal to this system, is a required basis. This system is orthonormal in $H$. For any $f \in A(F)$ the relation $\varphi_{i}^{\prime}(f)=0, \quad i \in \mathbb{N}$, implies that $e_{i}^{\prime}(f)=0, \quad i \in \mathbb{N}$, so the function $f$ vanishes on the set $F$ with all its derivatives, hence $f \equiv 0$ in a neighborhood of $F$. Therefore the system $\left\{\varphi_{i}\right\}$ is total and hence complete in the spaces (7.3), due to reflexivity all of them. Since $\varphi_{i}^{\prime}(f)=\left(f, \varphi_{i}\right)_{H}$, the orthogonal system $\left\{\varphi_{i}\right\}$ is maximal in $H$ and hence complete also in $H$.

From (7.7) we have that $\varphi_{i}^{\prime} \in A_{0}^{\perp}(F, \sigma)$ with $\sigma=\left(\left[\alpha_{\mu} s_{\alpha}(i)\right]+1\right)$. Therefore remembering that $\left\|\varphi_{i}^{\prime}\right\|=1$ we obtain, by Corollary 6.3 , the estimates

$$
\left|\varphi_{i}^{\prime}\right|_{\Omega_{\lambda}}^{*} \leq M \exp (\lambda+\delta) s_{\alpha}(i), \quad i \in \mathbb{N}, 0<\lambda<\infty
$$

with a constant $M=M(\lambda, \delta), \delta>0$.

On the other hand, due to $H \hookrightarrow A(\Omega)$, for each $\delta>0$ there is a constant $L=L(\delta)$ such that $\left|\varphi_{i}\right|_{\Omega_{\delta}} \leq L\left\|\varphi_{i}\right\|_{H}=L$ and $\varphi_{i} \in A_{0}\left((F, \sigma), \Omega_{\delta}\right)$ with $\sigma=$ $\left(\left[\alpha_{\mu} s_{\alpha}(i)\right]\right)$. Therefore, taking into account that $g_{\Omega_{\delta}}(F, \alpha ; z)=g_{\Omega}(F, \alpha ; z)+\delta$ and applying Theorem 6.2 , we obtain that the estimates

$$
\left|\varphi_{i}\right|_{\Omega_{\lambda}} \leq N \exp (-\lambda+\delta) s_{\alpha}(i), \quad i \in \mathbb{N}, \delta<\lambda<\infty,
$$

hold with some constant $N=N(\lambda, \delta), \delta>0$. The estimates (7.8) and (7.9) provide that, for each function $f$ belonging to any space from the list (7.3), its basis expansion $f(z)=\sum_{i \in \mathbb{N}} \varphi_{i}^{\prime}(f) \varphi_{i}(z)$ converges in the topology of that space.

The estimates (7.4) follows from (7.8), (7.9), taking into account the strict asymptotics

$$
s_{\alpha}(i) \sim \sigma_{n} i^{1 / n}, i \rightarrow \infty,
$$

which is derived from the evident strict asymptotics of the counting function:

$$
c(t):=\left|\left\{i: s_{\alpha}(i) \leq t\right\}\right| \sim \sum_{\mu=1}^{m} \frac{\left(\alpha_{\mu} t\right)^{n}}{n !}, t \rightarrow \infty .
$$




\section{Strict asymptotics for Kolmogorov diameters}

Now we are ready to prove the main results (Theorem 1.5 and Corollary 1.7). As it was emphasized in the introduction, an important part of the proof is covered by the following recent result of Nivoche-Poletsky (solving positively our conjecture from $[\mathbf{Z 6}, \mathbf{Z 7}]$; see Problem 1.4 above).

Proposition 8.1. Let $K$ be a compact set in Stein manifold $\Omega$ and $(K, \Omega)$ be a pluriregular pair. Then there exist a sequence of finite sets $F_{j}=\left\{\zeta_{\mu}^{(j)}: \mu=1, \ldots, m_{j}\right\} \subset$ $\Omega$ and a sequence $\alpha^{(j)}=\left(\alpha_{\mu}^{(j)}\right) \in \mathbb{R}^{m_{j}}, \alpha_{\mu}^{(j)}>0, \mu=1, \ldots, m_{j}$, such that the sequence $g_{\Omega}\left(F_{j}, \alpha^{(j)} ; z\right)$ converges to the function $\omega(\Omega, K ; z)-1$ uniformly on any compact subset of $\Omega \backslash K$.

Applying this result and our considerations developed in the previous sections, we prove now Theorem 1.5.

Proof. Let $\omega(z):=\omega(\Omega, K ; z)$. Take any pair of Hilbert spaces $\left(H_{0}, H_{1}\right)$ adherent to the pair $(A(K), A(\Omega))$, hence, by Theorem 4.12 , admissible for the couple $(K, \Omega))$. Since the strict asymptotics is the same for all admissible pairs of Banach spaces it is sufficient to prove that

$$
-\lim _{i \rightarrow \infty} \frac{\ln d_{i}\left(H_{1}, H_{0}\right)}{i^{1 / n}}=2 \pi\left(\frac{n !}{C(K, \Omega)}\right)^{1 / n} .
$$

First, by Theorem 4.11, the continuous linear imbeddings

$$
A\left(K_{\tau}\right) \hookrightarrow H_{0}^{1-\tau} H_{1}^{\tau} \hookrightarrow A\left(\Omega_{\tau}\right), 0<\tau<1,
$$

hold for the Hilbert scale $H_{\tau}:=H_{0}^{1-\tau} H_{1}^{\tau}$ with

$$
K_{\tau}:=\{z \in \Omega: \omega(z) \leq \tau\}, \Omega_{\tau}:=\{z \in \Omega: \omega(z)<\tau\} .
$$

Take some sequences $\varepsilon_{j} \downarrow 0$ and $\delta_{j} \downarrow 0$ so that

$$
\varepsilon_{j+1}<\varepsilon_{j}-2 \delta_{j}, j \in \mathbb{N} .
$$

By the Nivoche-Poletsky result (Proposition 8.1), for each $j \in \mathbb{N}$ there exists a finite set

$$
F^{(j)}=\left\{\zeta_{\mu}^{(j)}: \mu=1, \ldots, m_{j}\right\} \subset \Omega
$$

and a vector $\alpha^{(j)}=\left(\alpha_{\mu}^{(j)}\right) \in \mathbb{R}_{+}^{m_{j}}$ such that

$$
\left|g_{j}(z)-\omega(z)+1\right|<\delta_{j}, z \in K_{1-\varepsilon_{j}} \backslash \Omega_{\varepsilon_{j}},
$$

where $g_{j}(z):=g_{\Omega}\left(F^{(j)}, \alpha^{(j)} ; z\right), z \in \Omega$. Denote for $j \in \mathbb{N}$

$$
\Phi_{j}:=\left\{z \in \Omega: g_{j}(z) \leq-1+\varepsilon_{j}\right\}, D_{j}:=\left\{z \in \Omega: g_{j}(z)<-1+\varepsilon_{j}\right\}
$$

and consider the function

$$
\omega_{j}(z):=s\left(\Phi_{j}, \frac{g_{j}+1-\varepsilon_{j}}{1-\varepsilon_{j}} ; z\right)= \begin{cases}\frac{g_{j}(z)+1-\varepsilon_{j}}{1-\varepsilon_{j}}, & z \in \Omega \backslash \Phi_{j} \\ 0, & z \in \Phi_{j}\end{cases}
$$

It is clear that $\omega_{j}(z)=\omega\left(\Omega, \Phi_{j} ; z\right), z \in \Omega$. We show that

$$
\omega_{j}(z) \uparrow \omega(z), z \in \Omega \text {. }
$$


KOLMOGOROV PROBLEM ON WIDTHS ASYMPTOTICS AND PLURIPOTENTIAL THEORY1

Indeed, taking into account $(8.3),(8.4),(8.5),(8.6),(8.7)$, we get the inclusions:

$$
K_{\varepsilon_{j+1}-\delta_{j+1}} \subset \Phi_{j+1} \subset \Omega_{\varepsilon_{j+1}+\delta_{j+1}} \subset K_{\varepsilon_{j}-\delta_{j}} \subset D_{j} \subset \Phi_{j} \subset \Omega_{\varepsilon_{j}+\delta_{j}}
$$

Therefore the sequence $\omega_{j}(z)$ is non-decreasing and

$$
\omega\left(\Omega, K_{\varepsilon_{j}-\delta_{j}} ; z\right) \leq \omega_{j+1}(z) \leq \omega\left(\Omega, K_{\varepsilon_{j+1}-\delta_{j+1}} ; z\right), j \in \mathbb{N} .
$$

So (8.8) is proved.

Due to [BT1, BT2], we have

$$
C\left(\Phi_{j}, \Omega\right):=\int\left(d d^{c} \omega_{j}(z)\right)^{n} \downarrow \int\left(d d^{c} \omega(z)\right)^{n}=: C(K, \Omega) .
$$

On the other hand, due to (5.17),(5.18),

$$
C\left(\Phi_{j}, \Omega\right)=\left(\frac{2 \pi}{1-\varepsilon_{j}}\right)^{n} \sum_{\mu=1}^{m_{j}}\left(\alpha_{j, \mu}\right)^{n} .
$$

Given $\varepsilon>0$, due to (8.11), we can choose $j \in \mathbb{N}$ so that

$$
C(K, \Omega) \leq C\left(\Phi_{j}, \Omega\right) \leq(1+\varepsilon) C(K, \Omega) \text { and } \varepsilon_{j}<\varepsilon .
$$

Now we consider the basis $\left\{\varphi_{i}\right\}$ from Theorem 7.1 with the Hilbert space $H=H_{1}$ chosen in the beginning of the proof and with $F=F^{(j)}, \alpha=\alpha^{(j)}$. Let $G$ be the Hilbert space of all $x=\sum_{i \in \mathbb{N}} \xi_{i} \varphi_{i}$ such that

$$
\|x\|_{G}:=\left(\sum_{i \in \mathbb{N}}\left|\xi_{i}\right|^{2} \exp 2 \sigma_{n}\left(-1+\varepsilon_{j}\right) i^{1 / n}\right)^{1 / 2}<\infty,
$$

where, due to 8.12 ,

$$
\sigma_{n}=\left(\frac{n !}{\sum_{\mu=1}^{m_{j}}\left(\alpha_{j, \mu}\right)^{n}}\right)^{1 / n}=\frac{2 \pi}{1-\varepsilon_{j}}\left(\frac{n !}{C\left(\Phi_{j}, \Omega\right)}\right)^{1 / n} .
$$

By (2.11), (8.14) we have

$$
d_{i-1}\left(H_{1}, G\right)=\exp \sigma_{n}\left(-1+\varepsilon_{j}\right) i^{1 / n}
$$

which together with (8.13), (8.15) implies that

$$
2 \pi\left(\frac{n !}{(1+\varepsilon) C(K, \Omega)}\right)^{1 / n} \leq-\frac{\ln d_{i-1}\left(H_{1}, G\right)}{i^{1 / n}} \leq 2 \pi\left(\frac{n !}{C(K, \Omega)}\right)^{1 / n}
$$

Due to the estimates (7.4), (8.2), we get the imbeddings:

$$
H_{\varepsilon_{j}+\delta_{j}} \hookrightarrow A\left(\Omega_{\varepsilon_{j}+\delta_{j}}\right) \hookrightarrow A\left(\Phi_{j}\right) \hookrightarrow G \hookrightarrow A\left(D_{j}\right) \hookrightarrow A(K) \hookrightarrow H_{0} .
$$

Hence, by Proposition 2.1, there is a constant $M>0$ such that

$$
\frac{1}{M} d_{i}\left(H_{1}, H_{0}\right) \leq d_{i}\left(H_{1}, G\right) \leq M d_{i}\left(H_{1}, H_{\varepsilon_{j}+\delta_{j}}\right) .
$$

From the left inequalities in (8.17) and (8.18) we obtain the estimate:

$$
-\liminf _{i \rightarrow \infty} \frac{\ln d_{i}\left(H_{1}, H_{0}\right)}{i^{1 / n}} \geq 2 \pi\left(\frac{n !}{(1+\varepsilon) C(K, \Omega)}\right)^{1 / n} .
$$


Applying (2.12) with $\alpha_{1}=1$ and $\alpha_{0}=\varepsilon_{j}+\delta_{j}$, we derive from the right inequalities in (8.17) and (8.18) that

$$
-\limsup _{i \rightarrow \infty} \frac{\ln d_{i}\left(H_{1}, H_{0}\right)}{i^{1 / n}} \leq \frac{2 \pi}{1-2 \varepsilon}\left(\frac{n !}{C(K, \Omega)}\right)^{1 / n} .
$$

Since $\varepsilon>0$ is arbitrary, we conclude from (8.19),(8.20) that (8.1) is true. This completes the proof.

\section{Final remarks and some problems}

The following sufficient condition for the asymptotics (1.7) flows out from Proposition 4.6 and Corollary 4.13.

Corollary 9.1. Let $D$ be strongly pluriregular. Then the strict asymptotics (1.7) holds for any compact set $K$ making up a pluriregular pair with $D$.

Some more general sufficient condition (covering the one-dimensional Proposition 1.1) is represented by

Proposition 9.2. Let $(K, D)$ be a pluriregular pair on a Stein manifold, such that $D$ is an intersection of a countable decreasing sequence of open sets $D_{s}$ such that $H^{\infty}\left(D_{s}\right)$ is adherent to $A\left(D_{s}\right)$ for each $s$. Then $H^{\infty}(D)$ is adherent to $A(D)$ and the asymptotics (1.7) holds.

Whereas the pluripotential $\omega(D, K ; z)$ is an appropriate tool for the modified Kolmogorov problem (see, Theorem 1.5), for the solution of the original problem of Kolmogorov (1.3) in general case one needs to deal with another extremal function (see, e.g., $[\mathbf{Z 3}, \mathbf{S i b}])$ :

$$
\gamma(D, K ; z):=\limsup _{\zeta \rightarrow z} \sup \{u(\zeta): u \in \mathcal{A}(D, K)\}
$$

here $\mathcal{A}(D, K)$ consists of all functions $u(z)=\alpha \ln |f(z)|$ with $\alpha>0, f \in H^{\infty}(D)$ and $\left.u\right|_{K} \leq 0, u(z)<1$ in $D$. This is supported by the observation that in the conditions of Corollary 9.1 or Proposition 9.2 we have $([\mathbf{Z 3}, \mathbf{Z 6}, \mathbf{Z 7}])$ :

$$
\gamma(D, K ; z)=\omega(D, K ; z), \quad z \in D,
$$

and by the following result.

Theorem 9.3. Let the space $H^{\infty}(D)$ be adherent to $A(D)$. Then (9.2) holds for any compact set $K \subset D$ making up a pluriregular pair $(K, D)$.

Proof. The space $X_{0}=A C(K)$ is adherent to $A(K)([\mathbf{Z 3}])$ and $X_{1}=$ $H^{\infty}(D)$ is adherent to $A(D)$ by the assumption. Therefore, by Theorem 4.11, for any regular normal scale $X_{\alpha}$, connecting $X_{0}$ and $X_{1}$, the imbedding

$$
A\left(K_{\alpha}\right) \hookrightarrow X_{\alpha}, \quad 0<\alpha<1,
$$

holds. Suppose now that (9.2) is not valid. Then, since the function $\gamma(D, K ; z)$ does not exceed $\omega(D, K ; z)$, there is a point $z_{0} \in D \backslash K$ such that $\beta:=\gamma\left(D, K ; z_{0}\right)<$ $\omega\left(D, K ; z_{0}\right)=: \alpha$. Take $\sigma: \beta<\sigma<\alpha$ and denote $\Delta_{\sigma}:=\left\{z \in D: \gamma\left(D, K ; z_{0}\right)<\sigma\right\}$. By the definition (9.1), the estimate

$$
|x|_{L} \leq\left(\|x\|_{X_{0}}\right)^{1-\sigma}\left(\|x\|_{X_{1}}\right)^{\sigma}, \quad x \in X_{1},
$$

holds for any compact set $L \subset \Delta_{\sigma}$. Therefore the imbedding $\widehat{X}_{\sigma} \hookrightarrow A\left(\Delta_{\sigma}\right)$ is true, where $\widehat{X}_{\theta}, 0 \leq \theta \leq 1$, is the maximal scale of means spanned by $X_{0}, X_{1}$ 
([KPS], IV, Lemma 2.6). Since any regular scale is almost imbedded into any scale ([KPS], IV.11, Corollary 3), we obtain from here and (9.3) that $A\left(K_{\alpha}\right) \hookrightarrow X_{\alpha} \hookrightarrow$ $\widehat{X}_{\sigma} \hookrightarrow A\left(\Delta_{\sigma}\right)$. Since $z_{0} \in K_{\alpha}$ is an interior point of $\Delta_{\sigma}$ we get that each germ $x \in A\left(K_{\alpha}\right)$ has an analytic extension onto some fixed neighborhood of the point $z_{0}$, which is impossible, because $K_{\alpha}$ is holomorphically convex with respect to $D$. This contradiction completes the proof.

Problem 9.4. Let $(K, D)$ be a pluriregular pair on a Stein manifold $\Omega$. Is the condition (9.2) sufficient for the adherence of $H^{\infty}(D)$ to $A(D)$ ?

Also, in connection with Theorem 4.12 and Problem 4.15, the following question arises.

Problem 9.5. Let $(K, D)$ be a pluriregular pair on a Stein manifold $\Omega$. Does the condition (9.2) characterize the asymptotics (1.3) with the constant $\sigma=2 \pi\left(\frac{n !}{C(K, D)}\right)^{1 / n}$ ?

In conclusion we consider some examples.

Example 9.6. Let $E \subset \mathbb{R} \subset \mathbb{C}$ be the standard Cantor set. Then for the domain $D:=\overline{\mathbb{C}} \backslash E$ the space $H^{\infty}(D)$ consists only of constants. Hence, for any pluriregular compact set $K \subset D$, we have that $d_{i}\left(A_{K}^{D}\right)=0, i=1,2, \ldots$ and the asymptotics (1.3) has no sense ("holds with $\sigma=+\infty$ "), while $1 / \tau(K, D)<\infty$.

We notice that in this case $\gamma(D, K ; z) \equiv 0$, whereas $\omega(D, K ; z)$ is strictly positive in $D \backslash K$.

ExAmple 9.7. Let $E$ be again the usual Cantor set and $G \supset E$ be any domain in $\mathbb{C}$ satisfying the conditions of Proposition 1.1. Then for any regular compact set $K \subset D:=G \backslash E$ the asymptotics (1.3) holds with the constant $\sigma=\frac{1}{\tau(K, G)}>\frac{1}{\tau(K, D)}$.

So, in this case the constant $\sigma$ is defined by $\omega(G, K ; z)$, which is the harmonic extension of the function $\gamma(D, K ; z)$ onto the domain $G$, obtained after removing of the non-polar portion $E \subset \partial D$ having zero analytic capacity.

EXAMPLE 9.8. Let $E$ be a compact set of positive length on a rectifiable curve in $\mathbb{C}$, having no portion of zero length, and $D:=\overline{\mathbb{C}} \backslash E$. Though, due to the positive solution of Denjoy conjecture (see, e.g., $[\mathbf{V e}]$ ), the space $H^{\infty}(D)$ is non-trivial it seems that there is no answer to the following questions (even in the simplest case of Cantor sets of positive Lebesgue measure):

(1) Is $H^{\infty}(D)$ adherent to $A(D)$ ?

(2) Is the equality (9.2) true with some (hence with any) pluriregular compact set $K \subset D$ such that $\widehat{K}_{D}=K$ ?

(3) Is the couple $\left(A C(K), H^{\infty}(D)\right)$ admissible for $(K, D)$ if $K$ is as above or, what is the same, does the asymptotics (1.6) hold in this case?

Example 9.9. Sibony $[\mathbf{S i b}]$ studied domains

$$
D=z=\left\{\left(z_{1}, z_{2}\right) \in \mathbb{C}^{2}:\left|z_{1}\right|<1,\left|z_{2}\right|<\exp \left(-V\left(z_{1}\right)\right)\right\},
$$

where $0 \leq V\left(z_{1}\right) \leq 1$ is a continuous subharmonic function of the open unit disc and is equal to 0 on a discrete sequence such that every point of the unit circle is the nontangential limit of one of its subsequences. He proved that any function 
$f \in H^{\infty}(D)$ can be extended onto the bidisc $\mathbb{U}^{2}=\left\{\left|z_{1}\right|<1,\left|z_{2}\right|<1\right\}$ preserving its uniform norm. Hence for any pluriregular compact set $K \subset D$ we obtain that

$$
\gamma(D, K ; z) \equiv \omega\left(\mathbb{U}^{2}, K ; z\right)<\omega(D, K ; z), z \in D \backslash K
$$

and the asymptotics (1.6) holds with $\sigma=2 \pi\left(\frac{n !}{C\left(K, \mathbb{U}^{2}\right)}\right)^{1 / n}$.

For an arbitrary pluriregular pair $(K, D)$ on a Stein manifold $\Omega$ we set

$$
\Gamma(K, D):=\frac{1}{(2 \pi)^{n}} \int_{K}\left(d d^{c} \gamma(D, K ; z)\right)^{n} .
$$

and conjecture here that, if $\gamma(D, K ; z)<\omega(D, K ; z), z \in D \backslash K$, the asymptotics (1.3) ought to hold with $\sigma=\left(\frac{n !}{\Gamma(K, D)}\right)^{1 / n}$. Therewith (1.3) has to be understood as $d_{i}\left(A_{K}^{D}\right)=\exp \left(-\frac{i^{1 / n}}{o(1)}\right)$, if $\Gamma(K, D)=0$.

\section{References}

[AG] N. Aronszajn, E. Gagliardo, Interpolation spaces and interpolation methods, Ann. Mat. Pura Appl. 68 (1965), 51-117.

[A] A. Aytuna, On Stein manifolds $M$ for which $O(M)$ is isomorphic to $O\left(\Delta^{n}\right)$ as Fréchet spaces, Manuscripta Math. 62 (1988), 297-315.

[ARZ] A. Aytuna, A. Rashkovskii, V. Zahariuta, Widths asymptotics for a pair of Reinhardt domains, Ann. Pol. Math. 78 (2002), 31-38.

[B] K. I. Babenko, On E-entropy of a class of analytic functions, Nauchn. Doklady Vyssh. Shkoly, 2 (1958), 9-16.

[BT1] E. Bedford, A. B. Taylor, The Dirichlet Problem for a complex Monge-Ampére equation, Inventions Math, 37 (1976),1-44.

[BT2] E. Bedford, A. B. Taylor, A new capacity for plurisubharmonic functions, Acta Math, 149 (1982), 1-40.

[CLN] S. S. Chern, Levine H.I., L. Nirenberg, Intrinsic norms on a complex manifold, Global Analysis (Papers in honor of K. Kodaira) Univ. of Tokyo Press, Tokyo 1969, 119-139.

[E1] V. D. Erokhin, On asymptotics of E-entropy of analytic functions, Doklady AN SSSR 120 (1958), 949-952.

[E2] V. D. Erokhin, On the best approximation of functions analytically extendable from a given continuum into a given domain, Uspehi Matem. Nauk 14 (1968), 91-132

[FM] S. D. Fisher, C. A. Micchelli, The $n$-width of sets of analytic functions, Duke Math. J. 47 (1980), 789-801.

[Hr] L. Hörmander, Complex Analysis in several complex variables, North Holland/American Elsevier, 1973

[Kl] M. Klimek, Pluripotential Theory, Clarendon Press, Oxford-NY-Tokyo, 1991.

[K1] A. N. Kolmogorov, Estimates of the minimal number of elements of $\epsilon$-nets in various functional spaces an their application to the problem on the representation of functions of several variables with superpositions of functions of lesser number of variables, Uspehi Matem. Nauk 10 (1955), 192-193.

[K2] A. N. Kolmogorov, Asymptotic characteristics of certain totally bounded metric spaces, Doklady AN SSSR 108 (1956), 585-589.

[KT] A. N. Kolmogorov, V. M. Tikhomirov, $\varepsilon$-entropy and $\varepsilon$-capacity of sets in functional spaces, Uspehi Matem. Nauk 14 (1959), 3-86.

[KPS] S. G. Krein, Y. I. Petunin, E. M. Semenov, Interpolation of Linear Operators, Translation of Mathematical Monographs 54, AMS, Providence-Rhode Island, 1980.

[Le] P. Lelong, Notions capacitaires et fonction le Green pluricomplex dans les espaces de Banach, C. R. Acad. Sci. Paris 305 Serie I (1987), 71-76.

[LT] A. L. Levin, V. M. Tikhomirov, On theorem of V. D. Erokhin, Russian Math. Surveys 23 (1968), 119-132.

[LP] J.-L. Lions, J. Peetre, Sur une classe d'espaces d'interpolation, Inst. Hautes Études Sci. Publ. Math. 19 (1964), 5-68. 
[MV] R. Meise, D. Vogt, Introduction to Functional Analysis, Clarendon Press, Oxford, 1997.

$[\mathrm{M}]$ B. S. Mityagin, Approximative dimension and bases in nuclear spaces, Russian Math. Survey 16 (1963), 59-127.

[Ng] T. V. Nguyen, Bases de Schauder dans certain espaces de functions holomorphes, Ann. Inst. Fourier, 22 (1972), 169-253.

[N1] S. Nivoche, Sur une conjecture de Zahariuta et un probléme de Kolmogorov, C. R. Acad. Sci. Paris, Sér. I Math. 333 (2001), 839-843.

[N2] S. Nivoche, Proof of the conjecture of Zahariuta concerning a problem of Kolmogorov on the E-entropy, Invent. Math. 158 (2004), 413-450.

[Pi] A. Pietsch, Nuclear locally convex spaces, Berlin-Heidelberg-New York, 1972.

[P] E. Poletsky, Approximation of plurisubharmonic functions by multipole Green functions, Trans. Amer. Math. Soc. 335 (2003), 1579-1591.

[Sa1] A. Sadullaev, Plurisubharmonic measures and capacity on complex manifolds, Uspehi Matem. Nauk 36 (1981), 53-105.

[Sa2] A. Sadullaev, Rational approximations and pluripolar sets, Matem. Sbornik 119 (161) (1982), 96-118.

[Sib] N. Sibony, Prolongement des fonctions holomorphes bornées et métrique de Caratheodory, Invent. Math. 29 (1975), 205-230.

[Si] J. Siciak, Extremal plurisubharmonic functions in $\mathbb{C}^{n}$, Ann. Pol. Math. 39 (1981), 175-211.

[S] N. Skiba, Extendable bases and $n$-diameters of some classes of functions analytic on a Riemann surfaces, Ph. D. Thesis, Rostov State University, Rostov-na-Donu, 1979.

[SZ] N. Skiba, V. Zahariuta, Asymptotics of Kolmogorov diameters for some classes of harmonic functions on spheroids, J. Appr. Theory 102 (2000), 175-188.

[T1] V. M. Tikhomirov, On $\varepsilon$-entropy of some classes of analytic functions, Doklady AN SSSR 117 (1957), 191-194

[T2] V. M. Tikhomirov, Some Problems of Approximation Theory, MGU, Moscow, 1976.

[Ve] J. Verdera, Removability, capacity and approximation, in "Complex Potential Theory", NATO ASI Series, Series C: Mathematical and Physical Sciences, v. 439, ed. P.M. Gauthier, Kluwer AP, 1994.

[V] A. G. Vitushkin, The complexity estimation of tabulation problems, Fizmatgiz, Moscow, 1959.

[Vg] D. Vogt, Eine Charakterisierung der Potenzreihenträume von endlichen Typ und ihre Folgerungen, Manuscripta Math. 37 (1982), 269-301.

[VW] D. Vogt, M.J. Wagner, Charakterisierung der Unterräume und Quotientenräume der nuklearen stabilen Potenzreihenträume von endlichen Typ, Studia Math. 70 (1981), 63-80.

[W] H. Widom, Rational approximation and n-dimensional diameter, JAT, 5 (1972), 169-253.

[Wl] J. Walsh, Continuity of envelopes of plurisubharmonic functions, J. Math. Mech. 18 (1968),143-148.

[Z1] V. Zahariuta, Continuable bases in spaces of analytic functions of one and several variables, Sibirsk. Mat. Zh., 19 (1967), 277-292.

[Z2] V. Zahariuta, Some linear topological invariants and isomorphism of tensor products of scale centers, Izv. Sev.-Kavkaz. Nauch. Centra 4 (1974), 62-64.

[Z3] V. Zahariuta, Extremal plurisubharmonic functions, Hilbert scales, and the isomorphism of spaces of analytic functions of several variables, I, II. Teor. Funkciü, Funkcional. Anal. i Priložen. 19 (1974), 133-157; ibid. 21 (1974), 65-83.

[Z4] V. Zahariuta, Extremal plurisubharmonic functions, orthogonal polynomials and Bernstein-Walsh theorem for analytic functions of several complex variables, Ann. Pol. Math. 33 (1976), 137-148.

[Z5] V. Zahariuta, Isomorphism of spaces of analytic functions, Sov. Math. Dokl. 22 (1980), 631-634.

[Z6] V. Zahariuta, Spaces of analytic functions and maximal plurisubharmonic functions, Dr. Sci. Thesis, Rostov State University, Rostov-na-Donu, 1984, 281pp.

[Z7] V. Zahariuta, Spaces of analytic functions and complex potential theory, in: Linear Topological Spaces and Complex Analysis 1 (1994), 74-146.

[ZS] V. Zahariuta, N. Skiba, Estimates of n-diameters of some classes of functions analytic on Riemann surfaces, Matem. zametki 19 (1976), 899-911.

[Ze1] A. Zeriahi, Capacité, constante de Čebyšev et polynômes orthogonaux associés a un compact de $\mathbb{C}^{n}$, Bull. Sc. Math., $2^{e}$ série 109 (1985), 325-335. 
[Ze2] A. Zeriahi, Fonction de Green pluricomplexe à pôle à l'infini sur un espace de Stein parabolique et applications, Math. Sc. 69 (1991), 89-126.

Faculty of Engineering and Natural Sciences, Sabanci University, 34956 Tuzla/Istanbul, TURKEY

Current address: Faculty of Engineering and Natural Sciences, Sabanci University, 34956 Tuzla/Istanbul, Turkey

E-mail address: zaha@sabanciuniv.edu 